\title{
Cardiac myocyte-secreted cAMP exerts paracrine action via adenosine receptor activation
}

\author{
Yassine Sassi, ${ }^{1}$ Andrea Ahles, ${ }^{1}$ Dong-Jiunn Jeffery Truong, ${ }^{1}$ Younis Baqi, ${ }^{2}$ Sang-Yong Lee, ${ }^{2}$ Britta Husse, ${ }^{3}$ Jean-Sébastien Hulot, ${ }^{4,5}$ \\ Ariana Foinquinos, ${ }^{6}$ Thomas Thum, ${ }^{6,7}$ Christa E. Müller, ${ }^{2}$ Andreas Dendorfer, ${ }^{3,8}$ Bernhard Laggerbauer, ${ }^{1}$ and Stefan Engelhardt ${ }^{1,8}$ \\ 'Institut für Pharmakologie und Toxikologie, Technische Universität München (TUM), Munich, Germany. 2Pharma-Zentrum Bonn, Pharmazeutisches Institut, Pharmazeutische Chemie I, Universität Bonn, \\ Bonn, Germany. ${ }^{3}$ Walter Brendel Centre of Experimental Medicine, Ludwig-Maximilians-Universität München, Munich, Germany. ${ }^{4}$ Université Pierre et Marie Curie-Paris 6, INSERM UMR S 956, Paris, France. \\ ${ }^{5}$ Cardiovascular Research Center, Mount Sinai School of Medicine, New York, New York, USA. ${ }^{\circ}$ Institute of Molecular and Translational Therapeutic Strategies (IMTTS), Hannover Medical School, Hannover, \\ Cermany. ${ }^{7}$ National Heart and Lung Institute, Imperial College London, London, United Kingdom. ${ }^{8}$ German Center for Cardiovascular Research (DZHK), partner site Munich Heart Alliance, Munich, Cermany.
}

\begin{abstract}
Acute stimulation of cardiac $\beta$-adrenoceptors is crucial to increasing cardiac function under stress; however, sustained $\beta$-adrenergic stimulation has been implicated in pathological myocardial remodeling and heart failure. Here, we have demonstrated that export of cAMP from cardiac myocytes is an intrinsic cardioprotective mechanism in response to cardiac stress. We report that infusion of cAMP into mice averted myocardial hypertrophy and fibrosis in a disease model of cardiac pressure overload. The protective effect of exogenous cAMP required adenosine receptor signaling. This observation led to the identification of a potent paracrine mechanism that is dependent on secreted cAMP. Specifically, FRET-based imaging of cAMP formation in primary cells and in myocardial tissue from murine hearts revealed that cardiomyocytes depend on the transporter ABCC4 to export CAMP as an extracellular signal. Extracellular cAMP, through its metabolite adenosine, reduced cardiomyocyte cAMP formation and hypertrophy by activating A1 adenosine receptors while delivering an antifibrotic signal to cardiac fibroblasts by $\mathrm{A} 2$ adenosine receptor activation. Together, our data reveal a paracrine role for secreted cAMP in intercellular signaling in the myocardium, and we postulate that secreted cAMP may also constitute an important signal in other tissues.
\end{abstract}

\section{Introduction}

During the fight-or-flight response, activation of the sympathetic nervous system leads to a release of adrenaline and noradrenaline, which mediate their effects through the activation of adrenoceptors (1). Within the heart, $\beta$-adrenergic receptors ( $\beta$ ARs) couple mainly to stimulatory $G$ proteins $\left(G_{s}\right)$, thereby activating adenylyl cyclase to generate the second messenger cAMP from ATP. Intracellular cAMP formation represents the strongest mechanism for increasing cardiac function, but continuous activation of the cAMP pathway can promote cardiac hypertrophy and fibrosis (i.e., myocardial remodeling) and thereby contribute to cardiac disease (2). To prevent such detrimental consequences of sustained cAMP signaling, the extent and duration of cAMP formation underlie feedback control mechanisms, which include desensitization of $\beta$ ARs or rapid degradation of cAMP (3). In addition, stimulated cells invest a substantial amount of energy into transporting cAMP outside cells, a process that is mediated in mammals by members of the group of ATP-binding cassette (ABC) transporters, in particular, ABCC4, ABCC5, and ABCC11 (also termed MRP4, MRP5, and MRP8, respectively) (4-7).

A pivotal finding was that infusion of extracellular cAMP into mice exerted physiological effects in the kidney (8). These responses are conferred by adenosine receptors (adenosine

Conflict of interest: The authors have declared that no conflict of interest exists. Submitted: November 26, 2013; Accepted: October 14, 2014.

Reference information: / Clin Invest. 2014;124(12):5385-5397. doi:10.1172/JCI74349. receptor $1\left[A_{1} R\right], A_{2 A} R, A_{2 B} R$, and $\left.A_{3} R\right)$, which are expressed in most organs (9). A potential link between cAMP in the bloodstream and these receptors on renal cells was postulated based on the knowledge that 2 enzymes, ectonucleotide pyrophosphatase/ phosphodiesterase 1 (ecto-PDE, alias ENPP1) and ectonucleotide 5 '-nucleotidase (NT5E, the enzyme that converts AMP to adenosine, also known as CD73), convert cAMP to AMP and AMP to adenosine, respectively (10).

Interestingly, the heart is also equipped with the protein repertoire to export intracellular cAMP (11) and the enzymes to metabolize it to adenosine (12). This raises the intriguing question of whether the myocardium, potentially exemplary for many tissues, provides for its own source of extracellular adenosine by secretion of cAMP and whether this cAMP promotes cell-to-cell communication within the same tissue.

We addressed these issues by combining experiments in vivo with optical and biochemical analysis in vitro. Our data suggest an important regulatory role of secreted cAMP that serves to dampen the detrimental consequences of prolonged $\beta A R-c A M P$ signaling.

\section{Results}

Extracellular cAMP prevents adrenergically induced cardiac hypertrophy and fibrosis. To test for a role of extracellular cAMP in the heart, we chose chronic adrenergic stimulation of mice as an in vivo model for cardiac hypertrophy and fibrosis. Ten-week-old C57BL/6 N mice were infused for 7 days with isoproterenol (Iso) ( $\beta \mathrm{AR}$ agonist) and phenylephrine (PE) ( $\alpha_{1}$-adrenoceptor agonist) 
(30 mg/kg/d each) in the presence or absence of cAMP (30 mg/ $\mathrm{kg} / \mathrm{d}$ ). After 7 days, animals were sacrificed for analysis of the hearts. Mice treated with Iso/PE alone developed cardiac hypertrophy and fibrosis (Figure 1, A-C). Importantly, cAMP infusion significantly prevented these structural changes, as determined by morphometry and histology (Figure 1, A-C) and by quantification of the mRNAs that encode $M y h 7$, Col1a2, and Col3a1 (Figure 1D and Supplemental Figure 1, A and B; supplemental material available online with this article; doi:10.1172/JCI74349DS1). The presence of extracellular cAMP reduced ${ }^{3} \mathrm{H}$-thymidine incorporation in cardiac fibroblasts (CFs) (Supplemental Figure 1C), indicating that cAMP impairs their activation. Apoptosis of cardiac cells, in contrast, was not significantly altered by exogenous cAMP (Supplemental Figure 2).

Since the heart, like other organs, is equipped with membrane-anchored ENPP1 and NT5E $(13,14)$, exogenously applied cAMP is expected to be rapidly degraded to adenosine, which, upon receptor activation, may have induced the observed effects. Support for this idea came from experiments in which we inhibited the first enzymatic step in cAMP metabolization, that is, the conversion of cAMP to AMP by ENPP1. We identified SYL-001 as a potent and highly selective inhibitor of ENPP1 $\left(\mathrm{K}_{\mathrm{i}} 26.9 \mathrm{nM}\right.$, human ENPP1, > 1000-fold selective versus other ectonucleotidases) (Supplemental Figure 3, A-C). The presence of SYL-001 prevented extracellular cAMP from exerting its antihypertrophic and antifibrotic effects in Iso/PE-treated mice (Supplemental Figure 4). Furthermore, the myocardium appears to be equipped with all components of an endogenous cAMP/adenosine pathway, since cAMP accumulated in the pericardial fluid of mice subjected to transverse aortic constriction (TAC) and since ENPP1 inhibition exacerbated cardiac myocyte (CM) hypertrophy and cardiac fibrosis induced by pressure overload (Supplemental Figure 3, D-F). Consistent with our findings, other studies reported that deficiency of ENPP1 or NT5E impaired cardiac function or cardioprotective mechanisms, respectively $(15,16)$.

To determine which adenosine receptor was involved in extracellular cAMP signaling, we applied adenosine receptor antagonists in vivo. PSB16-P, a specific antagonist for $\mathrm{A}_{1} \mathrm{R}$ (see refs. 17, 18 for this and following antagonists), abolished the antihypertrophic effect of extracellular cAMP in Iso/PE-treated mice (Figure $1, A^{-} \mathrm{C}$ ), whereas fibrosis remained suppressed (Figure 1, A-C). Another drug that we tested in parallel was MSX-3, an antagonist of $A_{2 A} R$. With this, we observed a reciprocal effect, that is, it failed to reduce cardiac hypertrophy, but inhibited the ability of cAMP to prevent cardiac fibrosis (Figure 1, A-C). Histological analysis of cardiac tissue confirmed that extracellular cAMP prevented Iso/PE-induced CM hypertrophy and that PSB16-P reverted this reduction (Figure 1, E and $\mathrm{F}$ ). Since $\mathrm{A}_{2 \mathrm{~A}} \mathrm{R}$ antagonization did not completely inhibit the cAMP effect on CFs, we suspected that receptor subtype $A_{2 B}$ may also contribute to extracellular cAMP/ adenosine actions (moreover, $A_{2 B} R$ shows significant expression in CFs and is upregulated upon myocardial preconditioning; ref. 16). Indeed, we obtained support showing that $A_{2 B} R$ shares signaling function on CFs with $A_{2 A} R$ (see below and Figure 2).

Adenosine receptor subtype-specific effects were also observed in isolated CMs. Antagonization of the $A_{1} R$ efficiently impeded the antihypertrophic effect of cAMP, thus restoring
Iso/PE-induced CM hypertrophy (Figure 1, G and $\mathrm{H}$ ). Antagonists for $\mathrm{A}_{2 \mathrm{~A}} \mathrm{R}$ (SCH-442416), $\mathrm{A}_{2 \mathrm{~B}} \mathrm{R}$ (PSB-1115), or $\mathrm{A}_{3} \mathrm{R}$ (VUF 5574, not used before since it is not applicable in vivo) did not elicit significant effects, consistent with a far lower expression of these receptors in CMs than of $\mathrm{A}_{1} \mathrm{R}$ (Figure 2, $\mathrm{A}$ and $\mathrm{E}$, and Supplemental Figure 5, A and B).

These data suggest that extracellular cAMP protects the heart from adrenergically induced hypertrophy and fibrosis and that this is mediated through its metabolite adenosine acting mainly on $\mathrm{CM} \mathrm{A}_{1} \mathrm{R}$ and $\mathrm{CF} \mathrm{A}_{2} \mathrm{R}$.

Exogenous CAMP confers diametral changes of intracellular $c A M P$ in CMs and CFs. A quantitative PCR (qPCR) analysis of adenosine receptor expression in primary rat neonatal CMs or CFs (purity of isolates $>90 \%$ each) revealed that the $A_{1} R$ subtype is virtually the exclusive adenosine receptor in CMs (Figure 2A), whereas $A_{2 A} R$ and $A_{2 B} R$ dominate in CFs (ref. 19 and Figure 2E). $A_{3} R$ expression was marginal in CMs and CFs (Figure 2, A and E). Similar results were obtained from adult mouse CMs (AMCMs) or adult mouse CFs (AMCFs) (Supplemental Figure 5, A and B).

We then determined in what way distinct adenosine receptor profiles of cardiac cells affect their response to exogenously added cAMP. Since adenosine receptors couple to $\mathrm{G}_{\mathrm{s}}$ that regulate the activity of adenylyl cyclase, quantitation of intracellular cAMP was chosen to determine the effects extracellular cAMP exerts through adenosine. Isolated CMs or CFs were infected with an adenoviral vector for the expression of a fluorescence resonance energy transfer-based (FRET-based) cAMP sensor, and FRET was measured under various conditions in real time. Intriguingly, intracellular cAMP formation in CMs induced by $\beta$-adrenergic stimulation (Iso) was efficiently prevented by exogenous cAMP (Figure 2, B-D, and Supplemental Video 1). This effect was blocked by an $A_{1} R$ antagonist (DPCPX, $100 \mathrm{nM}$ ) and by the nonspecific adenosine receptor antagonist (DPSPX, $10 \mathrm{nM})$, butnotbyantagonists against $\mathrm{A}_{2 \mathrm{~A}} \mathrm{R}(\mathrm{SCH}-442416,100 \mathrm{nM})$, $\mathrm{A}_{2 \mathrm{~B}} \mathrm{R}$ (PSB-1115, $500 \mathrm{nM}$ ), or $\mathrm{A}_{3} \mathrm{R}$ (VUF 5574, $100 \mathrm{nM}$ ) (Figure 2, $\mathrm{B}-\mathrm{D})$. Next, this experimental setup was applied to CFs. As above, $\beta$-adrenergic stimulation by Iso increased intracellular cAMP, but, in contrast to CMs, exogenous cAMP enhanced formation of intracellular cAMP (Figure 2, F-H, Supplemental Video 2, and Supplemental Figure 6A for concentration response curves). Antagonization of $A_{2 A} R$ or $A_{2 B} R$ prevented this response, whereas interference with $A_{1} R$ or $A_{3} R$ had no consequence (Figure 2, F-H). We conclude that both adenosine $\mathrm{A}_{2} \mathrm{R}$ subtypes are involved in transmitting the extracellular cAMP/adenosine pathway to CFs. These experiments demonstrate that exogenous cAMP lowers the levels of intracellular cAMP in CMs (through activation of $G_{i}$-coupled $A_{1} R$ ), whereas $C F s$ respond by raising intracellular cAMP (through activation of $G_{s}$-coupled $A_{2 A} R$ and $A_{2 B} R$ ).

Intercellular CM-CF cAMP crosstalk. The potent effect of exogenously added cAMP on myocardial cells prompted us to investigate whether myocardial cells secrete cAMP in functionally relevant amounts or whether other nucleotides may likewise accumulate extracellularly in response to adrenoceptor activation. Isolated CMs or CFs were incubated with an inhibitor that blocks ENPP1 activity (IBMX, $300 \mu \mathrm{M}$ ) to prevent degradation of extracellular cAMP to AMP, and extracellular cAMP was detected in a colorimetric assay. In these analyses, isolated 
CMs showed basal cAMP export activity, which increased 5-fold when Iso was present (Figure 3A). Isolated CFs showed substantially less cAMP efflux, both under basal and Iso-induced conditions (Figure $3 \mathrm{~A}$ ). This points toward $\mathrm{CMs}$ as the primary cardiac source of secreted cAMP. Of note, extracellular levels of ATP or cGMP did not change in response to Iso stimulation in CMs (Supplemental Figure 7), thus emphasizing the specific role of cAMP as an extracellular signal upon $\beta$ AR stimulation.

We then asked whether export of cAMP from isolated CMs under adrenergic stimulation is able to condition the growth medium such that it elicits an intracellular response when transferred to CFs (see schematic drawing in Figure 3B). Since the recipient cells must be insensitive to adrenergic stimulation, $\mathrm{CFs}$ were derived from mice with homozygous deletion of the $\beta_{1}$ - and $\beta_{2}$-adrenoceptors ( $A d r b 1^{-/} A d r b 2^{-/-}$mice; ref. 20). These $A d r b 1^{-/-} A d r b 2^{-/}$CFs were then infected with an adenovirus encoding the cAMP sensor. The "sensor fibroblasts" indeed did not show intracellular cAMP formation in the presence of Iso, in contrast to exogenously added cAMP, which promoted intracellular cAMP formation via the activity of type 2 adenosine receptors (Supplemental Figure 6, A and B). We then stimulated adult WT CMs with Iso (10 $\mu \mathrm{M}$ for 1 hour), and the cell-free medium (hereafter termed conditioned medium [coM]) was transferred to the sensor CFs (Figure 3B). FRET detection in single sensor fibroblasts showed that $\mathrm{COM}$ from Iso-treated CMs significantly increased intracellular cAMP formation in CFs (Figure 3, C-E). This response was sensitive to the AR antagonist DPSPX (10 nM), indicating that adenosine was involved in this signaling.

Since the ABCC transporter family comprises the only active cAMP exporters known in the mammalian system, an inhibitor (MK571, $50 \mu \mathrm{M}$ ) was applied to test for their contribution to signaling of cAMP secreted by CMs. Indeed, MK571 potently prevented Iso-treated CMs from activating CFs (Supplemental Figure 8). Primary candidates for cAMP export activity among $\mathrm{ABCC}$ proteins are $\mathrm{ABCC} 4$ and $\mathrm{ABCC} 5$, given that cardiac expression of $\mathrm{ABCC} 11$ (the third potential cyclic nucleotide exporter, see ref. 7) is low (21). Intriguingly, coM from Iso-stimulated $\mathrm{Abcc4}^{-/-} \mathrm{CMs}$ completely failed to induce intracellular cAMP formation in sensor fibroblasts (Figure 3, C-E). The extent of this effect indicates that ABCC4 has a prominent role in CM-cAMP export. The link between ABCC4 and catecholamine-induced cAMP efflux from CMs is further supported by the preferential expression of $A b c c 4$ in this cell type (Supplemental Figure 5C) and by the finding that $A b c c 4^{-/-}$mice showed exacerbated cardiac hypertrophy and fibrosis in response to catecholamine infusion (Supplemental Figure 9, A and B).

cAMP-dependent communication between cardiac cells in cocultures and intact cardiac tissue. To simulate more closely the cardiac tissue context, primary CMs were directly layered onto preadhered sensor fibroblasts ( $A d r b 1^{-/-} A d r b 2^{-/-}$with cAMP sensor; see Figure $4 \mathrm{~A})$. One day later, a fraction of these CMs had come into direct contact with the sensor fibroblasts, as judged by passive movements of the latter through their attachment to beating CMs. $\beta$-Adrenergic stimulation of the cocultured CMs induced an increase of intracellular cAMP in attached sensor fibroblasts, thus confirming the observations made in experiments using coM (Figure 4, B and C).
To investigate native cardiac tissue for cAMP-dependent communication between cells, we devised a near-vivo setup based on intact slices of myocardium that maintain organotypic functions for several days (22). Slices from hearts of 12-week-old mice were taken into culture, and sensor fibroblasts were transferred onto them. Confocal microscopic imaging showed embedding of sensor fibroblasts (green in Figure 4D) into the slices. FRET determination of intracellular cAMP formation in sensor fibroblasts revealed that they reacted to $\beta$-adrenergic stimulation of the myocardial slice (Figure 4, E and F). In contrast, $\beta$-adrenergic stimulation of $\mathrm{Abcc}^{-/-}$slices failed to induce a FRET response in integrated sensor fibroblasts (Figure 4, E and F), suggesting dependence of the observed effects on secreted cAMP. Released endogenous cAMP was also sufficient to inhibit contractility of myocardial slices. Treatment of slices from WT mice with an $\mathrm{A}_{1} \mathrm{R}$ antagonist (DPCPX, $100 \mathrm{nM}$ ) led to a significant increase of Iso-induced contractility (Supplemental Figure 9, C and D). This positive inotropic effect of $A_{1} R$ blockade was entirely abolished in myocardial slices deficient for $\mathrm{Abcc4^{-/ }}$ (Supplemental Figure 9, C and D). These data demonstrate a key role of secreted cAMP for intercellular communication in the myocardium and substantiate the critical involvement of ABCC4 in this regulatory mechanism. Together, our results demonstrate that $\beta$-adrenergic stimulation causes CMs to secrete cAMP as a source of adenosine, which then signals to CFs (preventing fibrosis) and to vicinal CMs (preventing hypertrophy) (Figure 5).

\section{Discussion}

The results of this study indicate that cAMP secreted into the extracellular space is an important paracrine factor in the myocardium. Upon $\beta$-adrenergic stimulation, CMs actively export their intracellular second messenger cAMP, which, through its metabolite adenosine, controls the development of cardiac hypertrophy and fibrosis.

We provide the first evidence, to our knowledge, of the cardioprotective effects extracellular cAMP has on the myocardium, and we resolve the physiological impact of secreted cAMP at the cellular and molecular level.

The diametrically opposing effects of extracellular cAMP observed in CMs and CFs are in agreement with a cardioprotective role of secreted CAMP (see Figure 5), since reduced intracellular cAMP levels (after $G_{i}$ inhibition of adenylyl cyclase) interfere with prohypertrophic signaling in CMs (23) and elevated intracellular cAMP concentrations prevent activation of CFs $(12,24)$.

Inspired by the early finding that systemic infusion of cAMP alters renal activity (8), exogenously added cAMP was shown to also elicit effects in various cells and tissues in vitro $(25,26)$, yet the physiological relevance of the latter remained unresolved. We provide several lines of evidence that the myocardium as a sympathetically innervated organ can supply extracellular cAMP to establish a local paracrine signal: first, increased cAMP efflux was detected after Iso stimulation of primary CMs in vitro (Figure 2A) and in the pericardial fluid of mice subjected to TAC (Supplemental Figure 3D). Second, Iso-stimulated CMs conditioned their medium to promote protective signaling in CFs, a mechanism that requires the CAMP exporter $\mathrm{ABCC} 4$ on $\mathrm{CMs}$ and functional adenosine receptors on CFs (Figure 3, D and E). And third, block- 
A

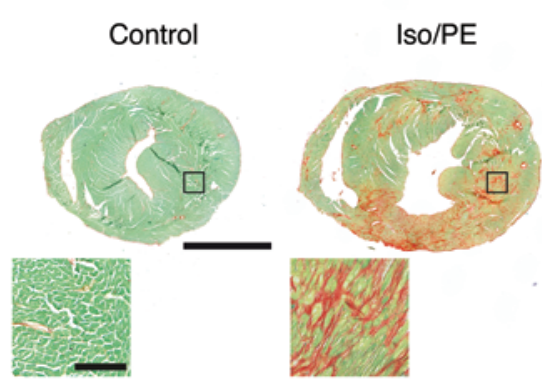

B

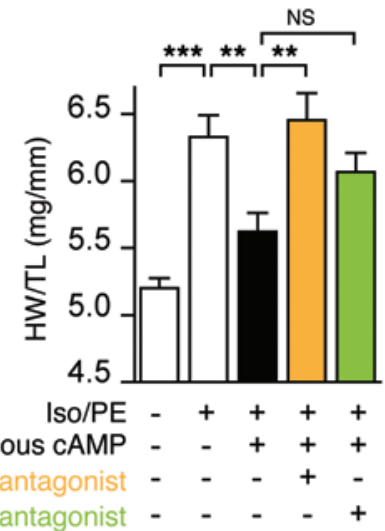

Iso/PE + exogenous cAMP

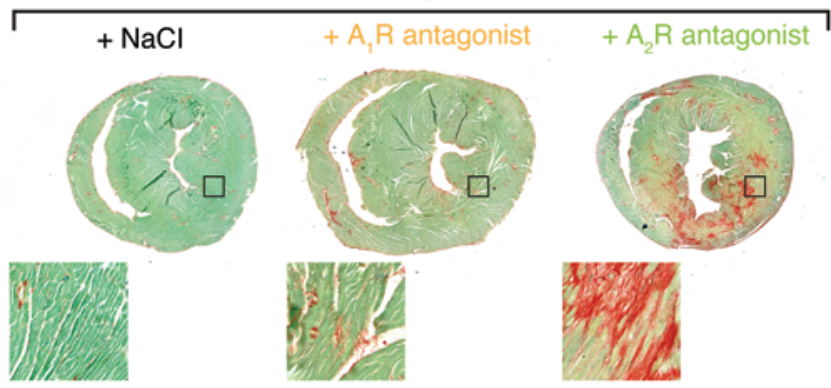

D

C

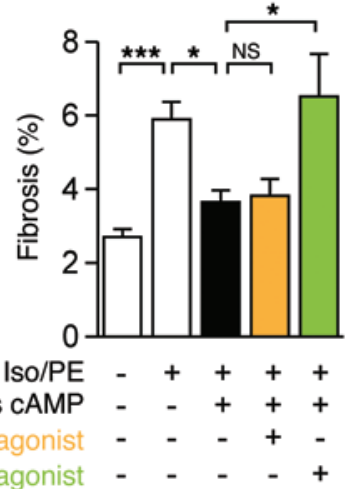

Iso/PE + exogenous cAMP

E

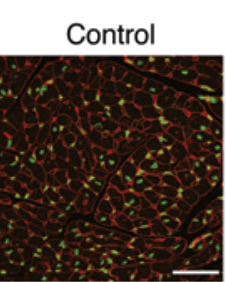

Iso/PE

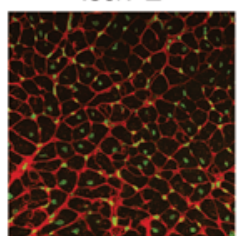

$+\mathrm{NaCl}$
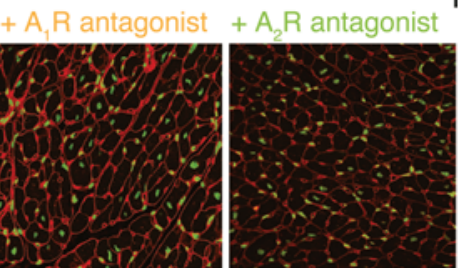

Iso/PE + exogenous CAMP
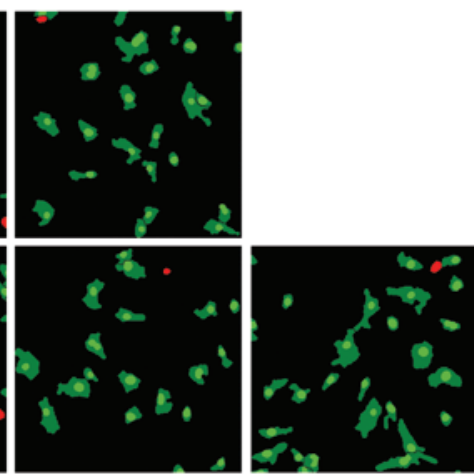

$+A_{1} R$ antagonist
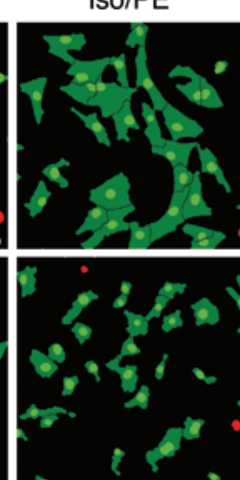

$+\mathrm{A}_{2 \mathrm{~A}} \mathrm{R}$ antagonist

Iso/PE + exogenous cAMP
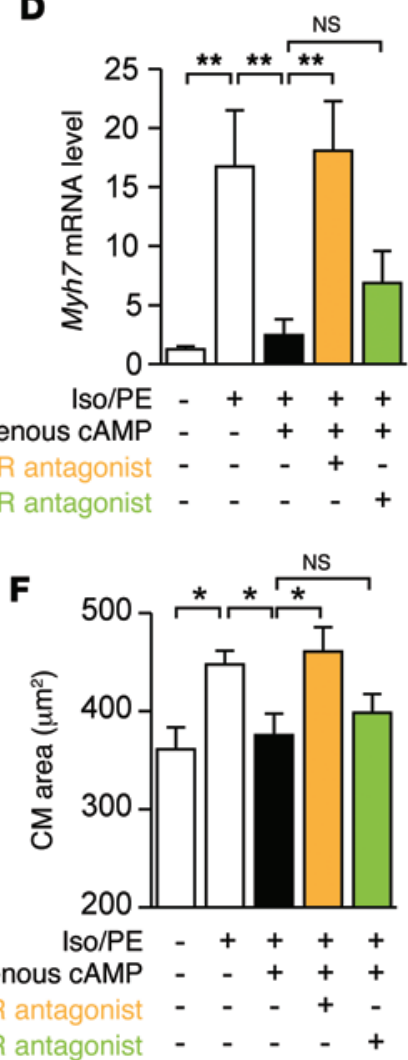

H

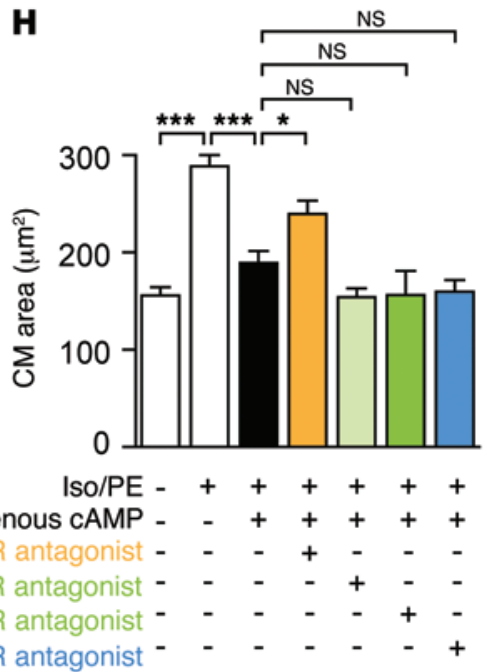


Figure 1. Extracellular cAMP prevents cardiac hypertrophy and fibrosis. (A-C) Mice were chronically infused with $1 \mathrm{so} / \mathrm{PE}$ (30 $\mathrm{mg} / \mathrm{kg} / \mathrm{d}$ each) and, where denoted, with exogenous CAMP (30 mg/kg/d), an $A_{1} R$ antagonist (PSB- $16 P, 5 \mathrm{mg} / \mathrm{kg} / \mathrm{d}$ ), or an $\mathrm{A}_{2 \mathrm{~A}} \mathrm{R}$ antagonist (MSX-3, $\left.5 \mathrm{mg} / \mathrm{kg} / \mathrm{d}\right)$ ). After 7 days, mice were sacrificed to assess cardiac remodeling. (A) Representative myocardial tissue sections after staining with Sirius Red (for collagen) and Fast Green counterstaining. Images at higher magnification are shown below. Scale bars: 2 mm (top row); $200 \mu$ m (bottom row). (B) Ratio of heart weight-to-tibia length (HW/TL) and (C) quantification of myocardial fibrosis. $n=9-16$ mice/group. (D) qPCR analysis of $\beta$-myosin heavy chain (Myh7) mRNA in myocardial tissue from the indicated groups. $n=5-9$ mice/group. (E) Representative WCA staining of left ventricle tissue after the indicated treatments and (F) quantitative analysis ( $n=6-8$ mice/group). Scale bar: $50 \mu \mathrm{m}$. (G and $\mathbf{H})$ NRCMs were treated with Iso/PE, and where denoted, cAMP (100 $\mu$ M) was added in the presence or absence of antagonists against $A_{1} R$ (DPCPX; $\left.100 \mathrm{nM}\right), A_{2 A} R(S C H-442416 ; 100 n M), A_{2 B} R$ (PSB-1115; 500 nM), or $A_{3} R$ (VUF-5574; 100 nM). (C) Processed images after immunofluorescent staining of primary CMs by an $\alpha$-actinin antibody (green cells). Nonmyocyte cells (marked in red) were defined as such if they yielded a DAPI signal, but not a signal for $\alpha$-actinin. Scale bar: $100 \mu \mathrm{m}$. (H) Quantitative analysis of the data ( $n=3$ experiments in triplicate). ${ }^{*} P<0.05 ;{ }^{* *} P<0.01 ;{ }^{* * *} P<0.001$.

ing the processing of endogenous, extracellular cAMP to AMP worsens TAC-induced cardiomyocytes hypertrophy and cardiac fibrosis (Supplemental Figure 3, E and F).

There is good reason to believe that cAMP signaling may be a common principle of many, if not all, mammalian tissues that are subject to sympathetic innervation. In support of this idea, extracellular cAMP levels have also been found to increase in adipose tissue in vivo as a response to exercise (27).

Is cAMP the only nucleotide-based source of extracellular signaling through adenosine receptors, and if others exist, are they relevant? Other candidates for paracrine signaling are ATP and cGMP $(28,29)$, but several aspects argue against a relevance for these nucleotides in the fight-or-flight response: first, release of ATP from CMs is reported to occur under stress conditions, such as hypoxia or mechanical stretch (28), but not during normal contraction (30). Under our experimental conditions, i.e., in the presence of Iso, we did not observe increased export of ATP or cGMP (Supplemental Figure 7). Furthermore, isolated CFs were recently shown to export ATP as a profibrotic signal accompanied by only a small degree of degradation to the antifibrotic adenosine (31). This suggests that $\beta$-adrenergic stimulation of the heart induces the secretion of cAMP as the primary source for the observed antifibrotic adenosine. Only severe conditions, such as myocardial ischemia, are expected to induce massive ATP efflux, which would then overrule the protective effect of cAMP (32-35). In this context, the question arises of how strong the protective effects of cAMP secretion are in disease conditions. Whereas infusion of cAMP into mice effectively prevented cardiac hypertrophy and fibrosis (Figure 1, B and C), an estimation on the potency of endogenous, extracellular cAMP relies on alternative approaches such as interference with ENPP1. The ENPP1-specific inhibitor SYL-001 exacerbated CM hypertrophy and cardiac fibrosis (Supplemental Figure 3, E and F), indicating that endogenous cAMP indeed confers cardioprotection.

Three mammalian cAMP transporters have thus far been identified, yet it seems doubtful that each of them has contributed to our experimental findings or that they are equally important for cAMP secretion in the human heart. Currently, there is no evidence for an ortholog of ABCC11 (MRP8) in mice, and in humans, no evidence of significant $A b c c 11$ expression in the heart exists. ABCC5 was not detected in CMs (11), whereas ABCC4 is markedly expressed in this cell type (ref. 11 and Supplemental Figure 5C). Also, genetic deletion of $A b c c 4$ alone was sufficient to completely block cAMP-based signaling outside the cell (Figure 3, C-E). This corresponds well with cardiac effects of this genotype in vivo (11) and indicates a prominent role of $\mathrm{ABCC} 4$ in the communication between $\mathrm{CMs}$ and $\mathrm{CFs}$.
The data presented here agree well with the antihypertrophic effect of adenosine (36) and $A_{1} R$ activation (23). In line with this, overexpression of $A_{1} R$ protected mice against myocardial infarction (37). Individual studies on both $\mathrm{A}_{2} \mathrm{Rs}$ point toward a cardioprotective role (32-34) through vasodilation and immunostimulation $(32,33)$ or the reduction of cardiac fibrosis after myocardial infarction $(32,35)$. With respect to the latter, it was shown that extracellular adenosine accumulation was inversely correlated with $\mathrm{CF}$ proliferation in vitro (38). Our findings now substantiate a functional link between cAMP secretion and $\mathrm{A}_{2} \mathrm{R}$ activation, based on the following: (a) secreted cAMP has an antifibrotic effect on the heart, (b) antagonists against $\mathrm{A}_{2} \mathrm{R}$ interfere with this effect in vivo, and (c) this subtype induces intracellular cAMP formation in CFs (thus activating antifibrotic pathways).

The protective effects of $A_{1} R$ and $A_{2} R$ agonists and early approval of adenosine as an antiarrhythmic drug supported a series of agonist-based clinical studies $(18,39)$. Our results also contribute to our understanding of the therapeutic efficacy of adenosine receptor agonists. By mimicking the action of CM-derived cAMP, these drugs exploit an intrinsic mechanism that protects the myocardium from the detrimental consequences of prolonged $\beta A R$ stimulation. Since earlier studies had indicated that exogenously added cAMP can alter the morphology or physiology of other organs (40), cell-to-cell communication via cAMP may be of broader relevance and represent a common principle.

\section{Methods}

Reagents. ZM-241385, PSB-1115, SCH-442416 and POM-1 were purchased from Tocris Biosciences. 2-( $N$-cyclohexylamino)ethanesulfonic acid (CHES), HEPES, and Tris were obtained from Applichem. Disodium hydrogenphosphate was purchased from Carl Roth. 4-Aminoantipyrine, AMP, ATP, calcium chloride, choline oxidase, DMSO, p-nitrophenyl phosphate, magnesium chloride, 1-oleoyl-sn-glycero3-phosphocholine (LPC) (18:1), peroxidase from horseradish, sodium chloride, sodium dodecyl sulphate (SDS), sodium hydroxide, sodium tetraborate (borax), 3-(N-ethyl-3-methylanilino)-2-hydroxypropanesulfonic acid (TOOS), and uridine were obtained from Sigma-Aldrich. Human recombinant soluble ENTPDase3 and human recombinant soluble alkaline phosphatase (TNAP), expressed in NSO cells from murine myeloma, were obtained from R\&D Systems GmbH. Human recombinant soluble ENTPDase1 and -2, and human recombinant soluble NT5E, expressed in CHO cells, were also obtained from R\&D Systems GmbH. Human recombinant soluble ENPP1-3 was expressed in Sf9 cells. The synthesis of PSB-16P (see Supplemental Figure 10 for structural information and numbering) started from 3-propyl-5cyclopentanecarboxamide-6-aminouracil (41), which was obtained 
A

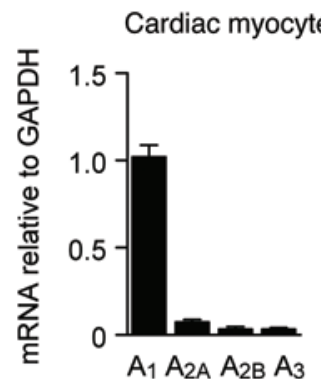

Adenosine receptor subtype

C

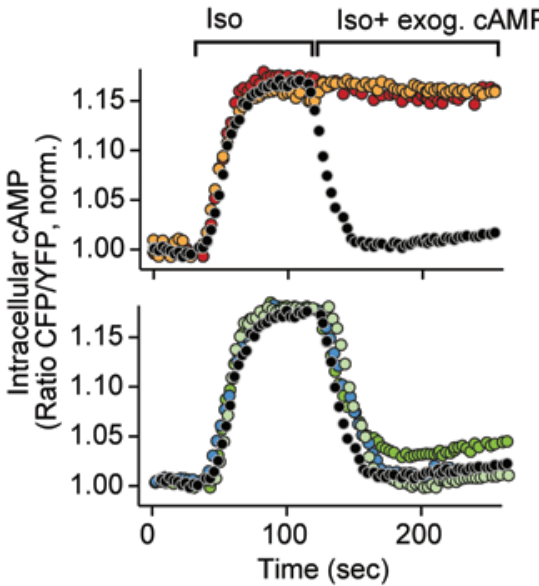

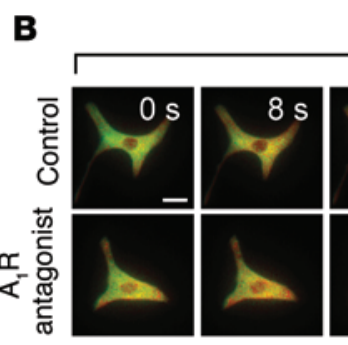

Iso
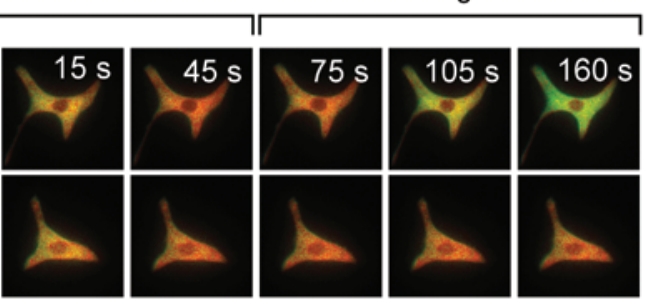

Intrac. CAMP

High

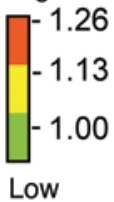

E

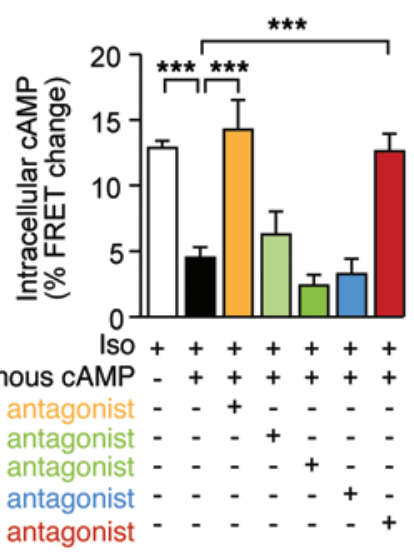

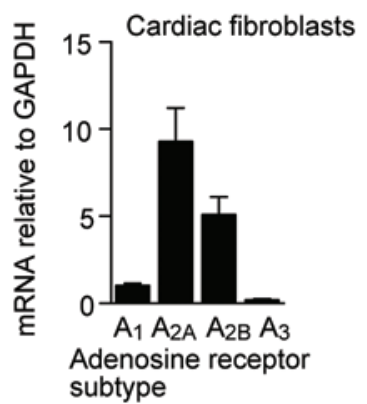

subtype
$\mathbf{F}$

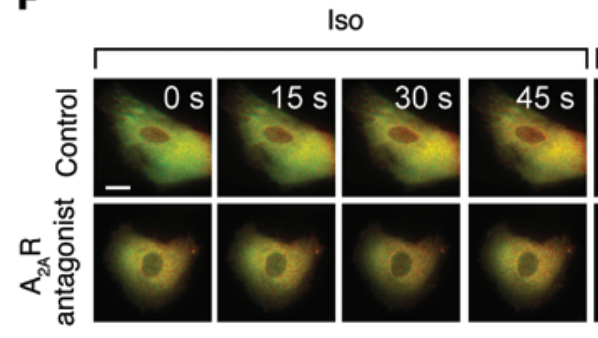

H

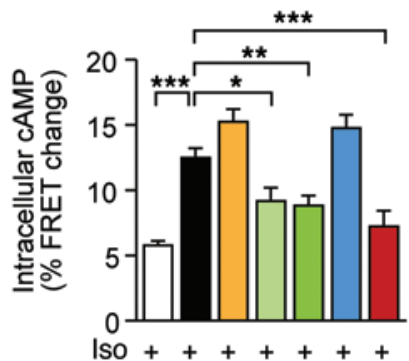

Exogenous CAMP -++++++

$A, R$ antagonist - $-++-\cdot$

$A_{A A} R$ antagonist - - - + - -

$\mathrm{A}_{2 \mathrm{~B}}^{2 \mathrm{~A}} \mathrm{R}$ antagonist - - - + +

$A R$ antagonist - - - - + +

AR antagonist - - - - - +
Intrac. CAMP
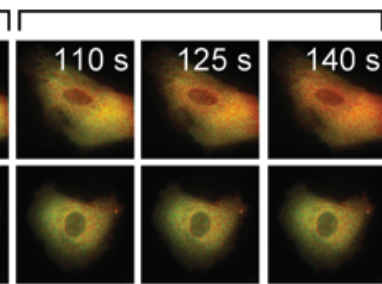

High

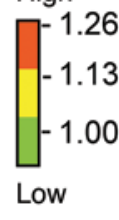

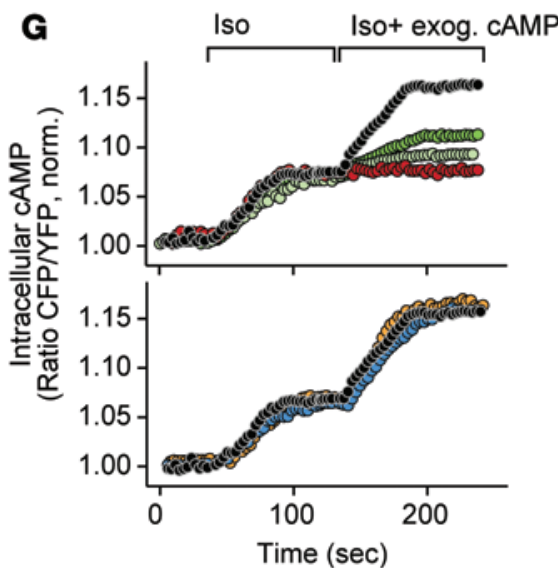

Figure 2. Exogenous cAMP confers opposing changes of intracellular CAMP in CMs and CFs. (A) Quantification of endogenous adenosine receptor subtype expression in NRCMs (by qPCR, $n=3$ ). (B) Real-time measurement of intracellular (intrac.) cAMP formation (shown as CFP/YFP ratio) in NRCMs transduced with a FRET-based sensor for CAMP. Cells were stimulated with Iso and CAMP in the absence (top row) or presence (bottom row) of the $A_{1} R$ antagonist DPCPX (100 nM). Scale bar: $10 \mu \mathrm{m}$. (C) Intracellular CAMP formation in NRCMs in the presence of Iso, with further addition of cAMP to the medium (100 $\mu \mathrm{M}$, black), and in the absence or presence of antagonists against $A_{1} R$ ( $100 \mathrm{nM} D P C P X$, yellow), $A_{2 A} R$ (100 nM SCH-442416, green), $A_{2 B} R$ (500 nM PSB1115 , green), $A_{3} R(100 n M$ VUF-5574, blue), and the nonspecific adenosine receptor antagonist DPSPX (10 nM, red). Representative tracings for the different treatment groups. (D) Quantitative analysis of the results. (E) Quantification of endogenous adenosine receptor subtype expression in isolated NRCF (by qPCR, $n=4,2$ days of culture). (F-H) Data derived from NRCF in an order analogous to that in the series above. In NRCF, exogenously added cAMP promoted the formation of intracellular cAMP ( $F$ and $\mathbf{G}$ ). Scale bar: $10 \mu \mathrm{m}$. Inhibition of $A_{2 A} R$ or of $A_{2 B} R$ (green tracings/bar) prevented the intracellular response to extracellular CAMP. (H) Quantitative FRET data were obtained from at least 9 cells/group. ${ }^{*} P<0.05 ;{ }^{*} P<0.01 ;{ }^{* *} P<0.001$. 
A

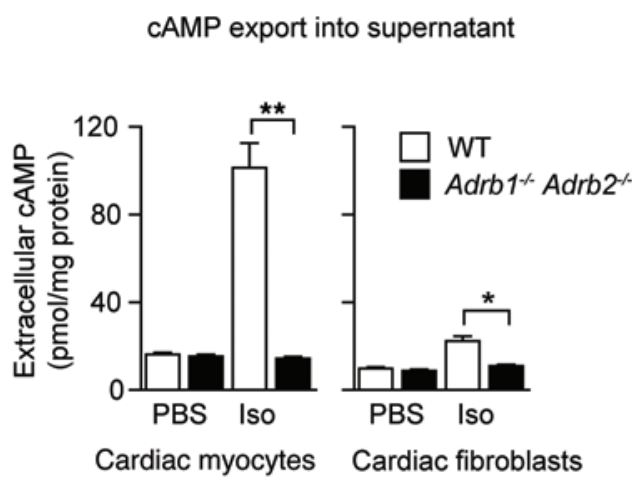

B

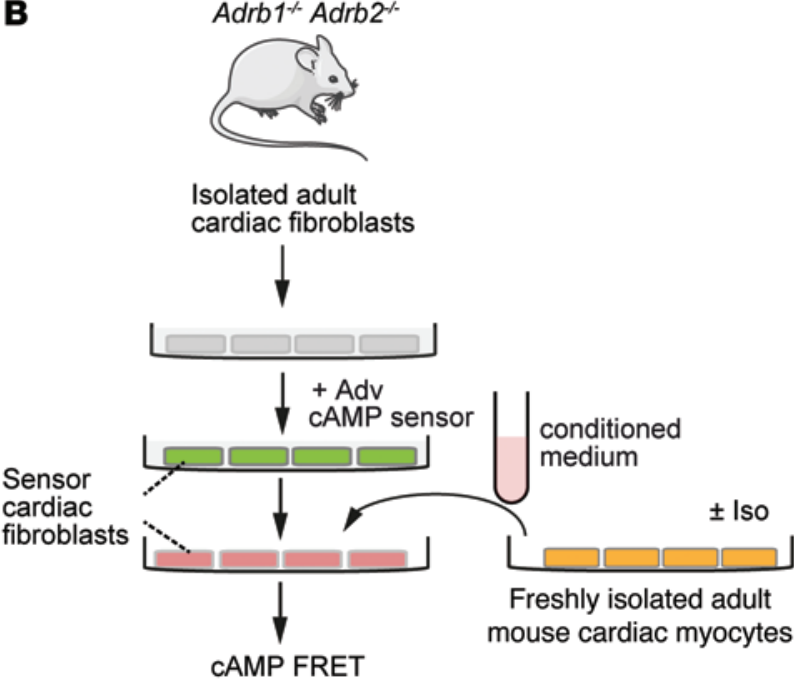

C

Sensor cardiac fibroblasts treated with supernatant from cardiac myocytes Iso-conditioned medium
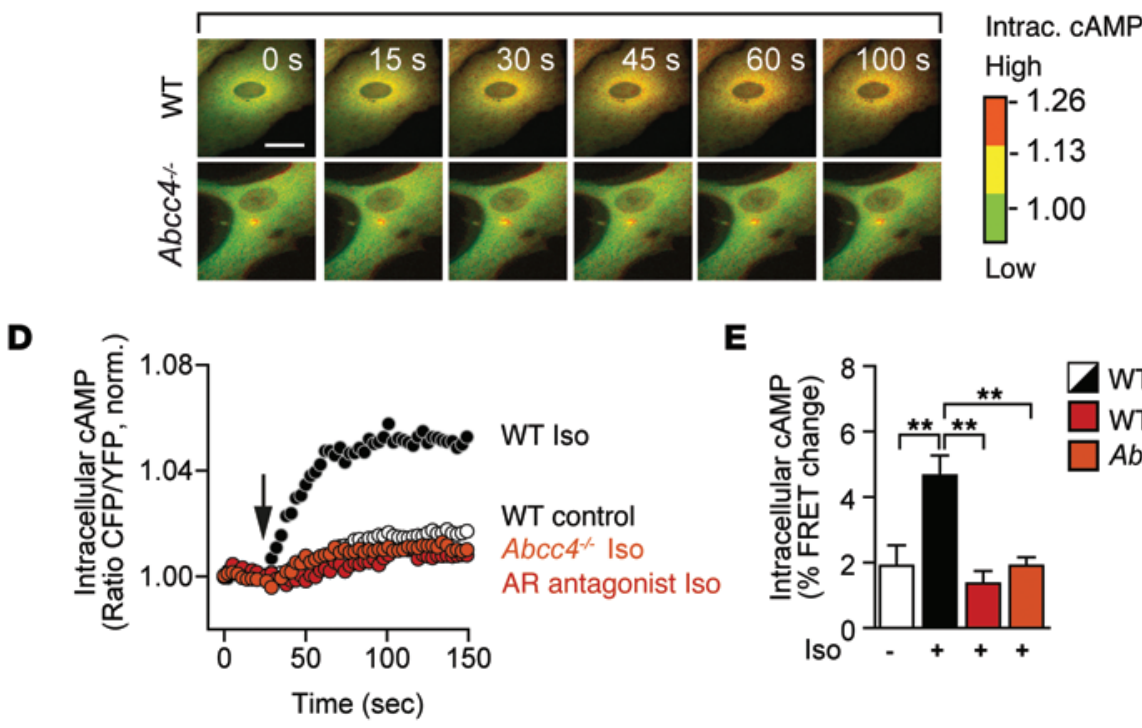

$\mathbf{E}$

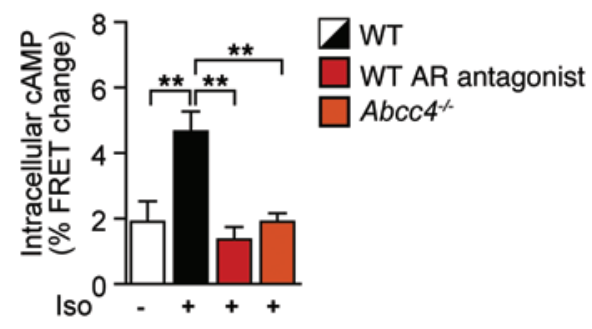

Figure 3. CM-derived secreted cAMP promotes intracellular CAMP formation in CFs. (A) Quantitation of extracellular cAMP in supernatants from cultured cardiomyocytes and CFs treated with $10 \mu \mathrm{M}$ Iso or PBS. CMs from mice deficient in $\beta_{1}$ - and $\beta_{2}$-adrenoceptors (Adrb1 $1^{-/-}$Adrb2 $2^{-/-}$) did not show Iso-induced extracellular cAMP accumulation. A phosphodiesterase inhibitor (IBMX, 300 $\mu \mathrm{M}$ ) was added to prevent cAMP degradation. $n=4-6$ experiments in duplicate. (B) FRET-based study design to assess cell-to-cell communication via cAMP. CFs from Adrb1 $1^{-1-}$ Adrb2 $2^{-/-}$mice (thus nonresponsive to Iso) were infected with an adenovirus encoding a FRET sensor for intracellular CAMP formation. To these CFs (green), coM from Iso-treated CMs (yellow) was added, and adenylyl cyclase activation in these CFs was monitored by the change in FRET signal of the CAMP sensor. (C-E) Intracellular CAMP formation in sensorinfected fibroblasts after addition of conditioned or control medium from WT and Abcc4-deficient CMs. (C) A time course of cAMP formation in CFs in the presence of coM from Iso-treated CMs with WT or Abcc4 ${ }^{-/-}$background. Scale bar: $10 \mu \mathrm{m}$. (D) Representative FRET recordings from CFs after incubation with coM from WT CMs without or with Iso treatment. Data shown in red or orange, respectively, were obtained using medium from CMs treated with Iso and the adenosine receptor antagonist DPSPX or from Iso-treated Abcc4 ${ }^{-/-}$CMs. Arrow indicates application of Iso or PBS. (E) Quantitative analysis of the results. $n=3-6$ experiments with $2-4$ cells each. ${ }^{* *} P<0.01$.

by standard procedures. Amide 1 was dissolved in dry dimethylformamide (DMF) in the presence of dry potassium carbonate, and alkylation in position 1 was performed with 3-iodo-1-propyl acetate as in previously described procedures (42). The subsequent ring closure to obtain xanthine derivative 3 was carried out in refluxing 1,1,1,3,3,3-hexamethyldisilazane (HMDS) in the presence of a catalytic amount of ammonium sulfate, followed by deacetylation in potassium hydroxide/methanol at room temperature to obtain compound 4 .
Finally, compound 4 was phosphorylated at the hydroxy group using phosphorus oxychloride in trimethyl phosphate (43). SYL-001 was identified as a potent and highly selective inhibitor of ENPP1 $\left(\mathrm{K}_{\mathrm{i}} 26.9\right.$ $\mathrm{nM}$, human ENPP1, > 1000-fold selective versus other ectonucleotidases). It displayed a noncompetitive mechanism of inhibition (for details, see Supplemental Figure 3, A-C). All other agonists, antagonists and inhibitors, and cAMP were purchased from Sigma-Aldrich. Reagents were dissolved as suggested by the manufacturer. 
A
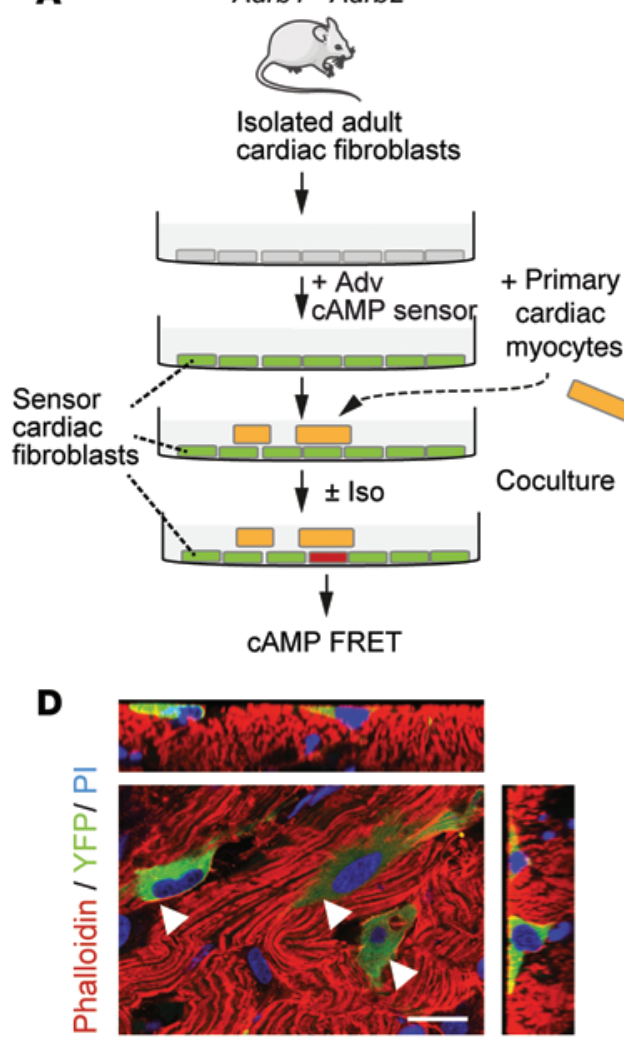

B

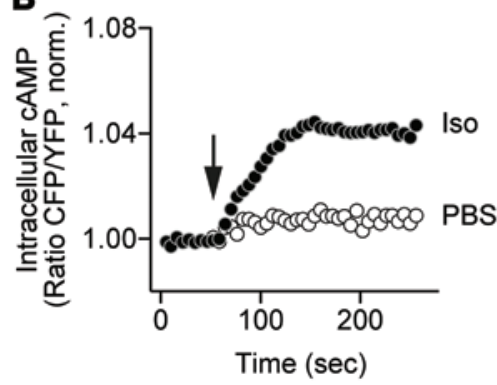

E

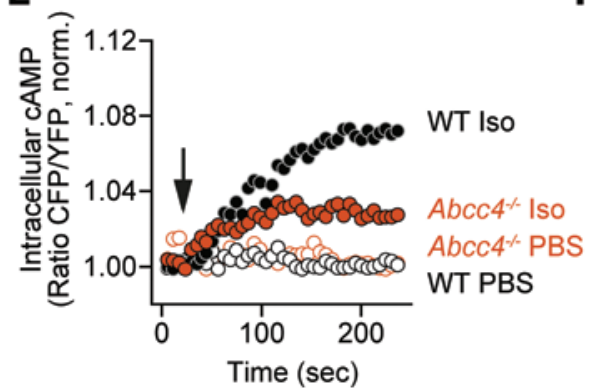

C

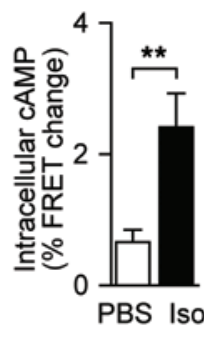

$\mathbf{F}$

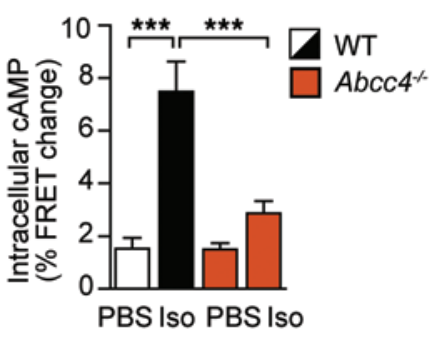

Figure 4. Intercellular communication through secreted cAMP between CMs and CFs in cocultures and living cardiac tissue. (A) Experimental scheme to assess the role of secreted CAMP in cocultures of CMs and CFs. Primary CFs from Adrb1 ${ }^{-1-}$ Adrb2 $2^{-1-}$ mice (green) were infected with an adenovirus for the expression of a FRET-CAMP sensor (termed sensor CFs). WT NRCMs (yellow) were plated on top of the cultured primary CFs. The response of CFs to CMderived cAMP (after stimulation with $10 \mu \mathrm{M}$ Iso) was monitored by FRET. (B) Representative FRET recordings in CFs after Iso-induced cAMP export from CMs. PBS served as a negative control. (C) Quantification of the results. $n=10-15$ cells (3-6 independent experiments). ${ }^{*} P<0.01$, determined by unpaired $t$ test with Welch's correction. (D) Multicolor 2-photon images of a slice from cultured mouse myocardium to which sensor CFs had been transplanted. Histochemical stainings were for phalloidin (red), adenovirus-encoded cAMP-sensor (YFP, green), and propidium iodide (PI, blue). Image perspectives are overhead (large), horizontal (top), and vertical (right) cross sections. Arrowheads indicate the addition of isoproterenol or PBS. Scale bar: $20 \mu \mathrm{m}$. (E) cAMP formation in individual CFs after incubation of WT myocardial slices with Iso (black circles) or PBS (white circles, black outline). Data from an analogous experiment with slices from Abcc4 $4^{-1-}$ mice are displayed in red (red circles, Iso; white circles, red outline, PBS). (F) Quantification of the results. $n=7-15$ cells from 3-5 independent experiments. ${ }^{* *} P<0.001$, NS, not significant versus WT; Iso determined by 2-way ANOVA with Bonferroni's post hoc analysis.

Animal models. As a model for chronic adrenergic stimulation, miniosmotic pumps (Alzet) containing (-)- Iso and PE (delivering $30 \mathrm{mg} / \mathrm{kg} / \mathrm{d}$ each) were implanted subcutaneously into 10-week-old FVB male mice (animals were randomly assigned with parallel group design). Animals were continuously infused for 7 days with Iso/PE alone or together with cAMP $(30 \mathrm{mg} / \mathrm{kg} / \mathrm{d})$, the $\mathrm{A}_{1} \mathrm{R}$ antagonist (PSB$16 \mathrm{P}, 5 \mathrm{mg} / \mathrm{kg} / \mathrm{d}$ ), the $\mathrm{A}_{2} \mathrm{R}$ antagonist (MSX-3, $5 \mathrm{mg} / \mathrm{kg} / \mathrm{d}$ ), the specific ENPP1 inhibitor (SYL-001, $10 \mathrm{mg} / \mathrm{kg} / \mathrm{d}$ ), or the ENPP1 and adenosine receptor antagonist (DPSPX $10 \mathrm{mg} / \mathrm{kg} / \mathrm{d}$ ). Then mice were sacrificed to determine parameters of cardiac hypertrophy and fibrosis. For the analysis of collagen deposition, paraffin sections of left ventricular myocardium were stained with Sirius Red and Fast Green. Collagen content was calculated as the percentage of the area in each section that was stained with Sirius Red. Thoracic aortic constriction was performed on 8-week-old male C57BL/6 N mice essentially as described previously (44). In sham surgery, only the chest was opened, but no ligation of the aorta was carried out.

$A b c c 4^{-/-}$mice were established by the John Schuetz laboratory (45) (St. Jude Children's Research Hospital, Memphis, Tennessee, USA) and repeatedly backcrossed to FVB mice to greater than $99 \%$ FVB (46). Adrb1 $1^{-/}$Adrb2 $2^{--}$mice were generated by Rohrer et al. (20). All genotypes were verified by PCR analysis.

Isolation of CMs and CFs from mouse and rat hearts. Neonatal rat CMs (NRCMs) and neonatal rat CFs (NRCFs) were isolated from 1- to 2-day-old Sprague-Dawley rats after decapitation, as described previously (47). Cells were cultured in MEM with 5\% FCS on uncoated culture dishes. For coculture experiments, NRCMs were layered onto preadhered $\mathrm{Adrb1^{-- }}$ Adrb2 $2^{--}$adult CFs that had been infected 48 hours earlier with the Adv cAMP sensor. AMCMs and AMCFs were isolated from WT, Adrb1 $1^{-/} A d r b 2^{-/-}$, or Abcc4 $4^{-/-}$mice. Briefly, hearts were extracted and coronary arteries were perfused with buffer A $(113 \mathrm{mM} \mathrm{NaCl}$, $4.7 \mathrm{mM} \mathrm{KCl}, 0.6 \mathrm{mM} \mathrm{KH}_{2} \mathrm{PO}_{4}, 0.6 \mathrm{mM} \mathrm{Na}_{2} \mathrm{HPO} 4,1.2 \mathrm{mM} \mathrm{MgSO}_{4}, 12$ $\mathrm{mM} \mathrm{NaHCO}, 10 \mathrm{mM} \mathrm{KHCO}_{3}, 10 \mathrm{mM}$ HEPES, and $30 \mathrm{mM}$ taurine) in a retrograde fashion by cannulation of the aorta. Collagenase type II (Worthington) was added to the buffer to enzymatically dissociate ventricular cells. The disintegrated tissue was allowed to settle for 10 minutes at $37^{\circ} \mathrm{C}$ before the $\mathrm{CF}$-enriched supernatant (supernatant A) and the pellet-containing CMs were resuspended in buffer B $(47.5 \mathrm{ml}$ 


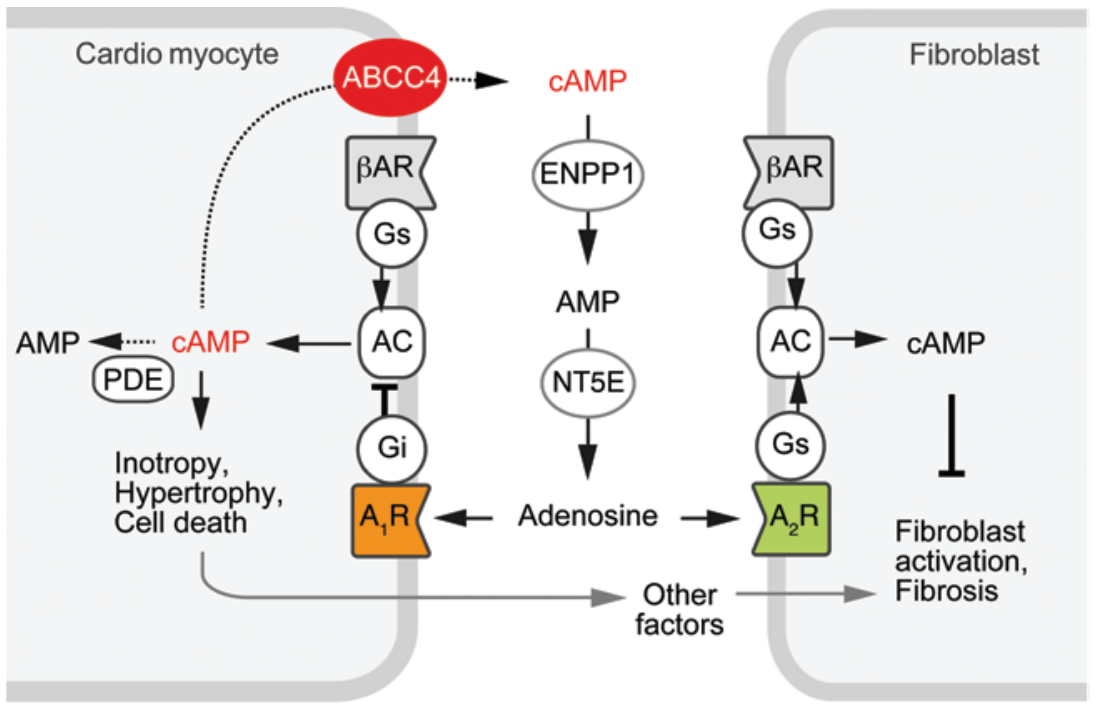

Figure 5. Model of signal transmission between adrenergically stimulated CMs and CFs through secreted cAMP. In CMs (left), stimulation of $\beta A R s$ activates adenylate cyclase $(A C)$ via $G_{s}$, causing rapid cAMP formation. This second messenger has the potential to elicit inotropic effects and CM hypertrophy and apoptosis. Alternatively, $A B C$ proteins, in particular ABCC4, export CAMP, which is stepwise metabolized by ENPP1 and NT5E to adenosine. Adenosine feeds back onto vicinal $\mathrm{CMs}$ through binding to its predominant adenosine receptor subtype, $A_{1} R$, thereby engaging $G i$ to inhibit intracellular CAMP formation. On CFs (right), adenosine activates its predominant receptor subtypes, $A_{2 A} R$ and $A_{2 B} R$. Their coupling to $G_{5}$ activates adenylate cyclase, thus enhancing cAMP formation, which, in this cell type, inhibits proliferation and extracellular matrix deposition (thus preventing cardiac fibrosis).

perfusion buffer A, $\left.2.5 \mathrm{ml} \mathrm{FCS}, 62.5 \mathrm{ml} 10 \mu \mathrm{M} \mathrm{CaCl}_{2}\right) \cdot \mathrm{CaCl}_{2}$ was gradually added back to yield a final concentration of $100 \mu \mathrm{M}$, and the isolated cells were preplated in MEM (5\% FCS, 10 mM 2,3-butanedione monoxime, $2 \mathrm{mM}$ L-glutamine, and $1 \%$ penicillin/streptomycin) for 1 hour at $37^{\circ} \mathrm{C}$ and $1 \% \mathrm{CO}_{2}$. For CFs, supernatant was centrifuged for 5 minutes $(225 g)$ and the pellet was resuspended in 5\% FCS MEM culture medium, followed by plating on a 6-cm culture dish.

Assessment of CM hypertrophy. NRCMs were plated onto optically optimized 96-well plates (ibidi) in MEM medium containing $1 \%$ FCS. Twenty-four hours later, the medium was exchanged to $0.1 \%$ FCS in MEM medium in the presence or absence of $50 \mu \mathrm{M} \mathrm{PE}$, $10 \mu \mathrm{M}$ Iso, $100 \mu \mathrm{M}$ cAMP, $100 \mathrm{nM}$ DPCPX, $100 \mathrm{nM}$ ZM-241385, or 100 nM VUF-5574. Forty-eight hours later, cells were washed twice with PBS and fixed for 10 minutes with paraformaldehyde (4\%). Immunostainings of NRCMs in 96-well format and automated cell size measurement were performed as described (48).

${ }^{3} \mathrm{H}$-Thymidine incorporation. Adult rat $\mathrm{CFs}$ were plated onto 48-well plates in MEM medium containing 1\% FCS. Twenty-four hours later, the medium was replaced with $10 \%$ FCS in MEM medium in the presence or absence of $100 \mu \mathrm{M}$ cAMP. After 24 hours, the treatments were repeated with freshly prepared solutions but supplemented with ${ }^{3} \mathrm{H}$-thymidine $(1 \mu \mathrm{Ci} / \mathrm{ml}$, Hartmann Analytic) for an additional 12 hours. Cells were then washed with PBS and incubated with $5 \%$ trichloroacetic acid for 1 hour at $4^{\circ} \mathrm{C}$. Cells were subsequently lysed in $0.5 \mathrm{M} \mathrm{NaOH}$ for 30 minutes at $37^{\circ} \mathrm{C}$, and lysates were mixed with $4 \mathrm{ml}$ scintillation fluid (Roth) for quantitation of ${ }^{3} \mathrm{H}$.

Quantification of cAMP, cGMP, or ATP release into culture medium. cAMP was measured in culture supernatants of isolated AMCMs and AMCFs by an enzymatic immunoassay as recommended by the manufacturer (cAMP parameter assay kit, R\&D Systems). Cells were seeded in a 6-well plate (coated with laminin in the case of AMCMs) and treated for 1 hour with Iso $(10 \mu \mathrm{M})$ and/or IBMX $(300 \mu \mathrm{M})$. After collection of the medium and centrifugation (1 minute, $100 \mathrm{~g}$ ), cAMP was quantified relative to total protein content of cells.

cAMP and cGMP were also measured in culture supernatants of isolated NRCMs and NRCFs, in addition to ATP quantification. Twenty-four hours after seeding, NRCMs and NRCFs were treated as above. cGMP was quantified by an enzymatic immunoassay as recom- mended by the manufacturer (cGMP parameter assay kit, R\&D Systems). Cultures dedicated to ATP quantification were supplemented with the ectonucleotidase inhibitor POM-1 $(30 \mu \mathrm{M}) 5$ minutes before quantification to prevent the degradation of ATP. After collection of the medium and centrifugation ( 1 minute, $100 \mathrm{~g}$ ), ATP was quantified as recommended by the manufacturer (ATP assay, Promega).

Quantification of cAMP in pericardial fluid. Miniosmotic pumps containing the ENPP1 inhibitor (SYL-001, $7.5 \mathrm{mg} / \mathrm{kg} / \mathrm{d}$ ) were implanted subcutaneously into 8-week-old FVB male mice. Two days later, the mice were subjected to sham or TAC surgeries. Twenty-four hours later, the pericardial fluid was collected as described previously (49). Briefly, the pericardium was incised and washed with $350 \mu \mathrm{l}$ PBS (containing SYL-001, $200 \mathrm{nM}$ ). The fluid was centrifuged (7800 $g$, $5 \mathrm{~min}$ utes) and aliquots were stored at $-80^{\circ} \mathrm{C}$. cAMP was quantified as above.

Assessment of intracellular cAMP formation by FRET. NRCMs,

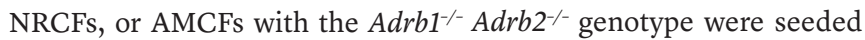
directly onto coverslips (in the case of CMs coated with poly-Dlysine). Twenty-four hours later, cells were infected with an adenovirus (MOI of 100) encoding a fusion construct in which the fluorophores yellow fluorescent protein (YFP) and cyan fluorescent protein (CFP) had been cloned at opposite ends of the cAMP-binding domain of Epac (50). Cells were transferred in buffer C (13 mM $7 \mathrm{NaCl}, 5.4 \mathrm{mM}$ $\mathrm{KCl}, 2 \mathrm{mM} \mathrm{CaCl}_{2}, 1 \mathrm{mM} \mathrm{MgCl}_{2}, 10 \mathrm{mM}$ HEPES, pH 7.3) to the experimental chamber at $37^{\circ} \mathrm{C}$, and images were taken in 3-second intervals on a Zeiss AxioObserver inverted microscope equipped with an oil immersion $\times 40$ objective, polychrome V light source (Till Photonics), and an Evolve-EM512 digital camera (Visitron Systems). FRET was monitored as the emission ratio at $535 \pm 20 \mathrm{~nm}$ and $480 \pm 15 \mathrm{~nm}$ upon excitation at $436 \pm 10 \mathrm{~nm}$ using MetaFluor software (Visitron Systems). Data were corrected for transmittance of CFP into the 535$\mathrm{nm}$ channel to provide a corrected CFP/YFP ratio. Agonist-induced FRET changes were recorded on cells under continuous exposition to buffer $\mathrm{C}$ plus Iso (10 $\mathrm{nM}$ in CMs and $1 \mathrm{nM}$ in CFs $) \pm \mathrm{cAMP}(100 \mu \mathrm{M}) \pm$ $\operatorname{DPCPX}(100 \mathrm{nM}) \pm \operatorname{DPSPX}(10 \mathrm{nM}) \pm \mathrm{SCH}-442416(100 \mathrm{nM}) \pm$ PSB$1115(500 \mathrm{nM}) \pm$ VUF-5574 $(100 \mathrm{nM})$. When adenosine antagonists were used, cells were pretreated 10 minutes before measurements.

Assessment of intercellular cAMP signaling by transfer of coM. To test which effects cAMP exported from CMs has on CFs, cells were 
isolated from adult mouse hearts, either from WT or the $\mathrm{Abcc4}^{-1-}$

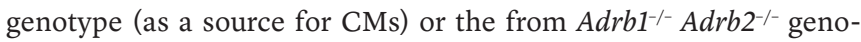
type (for CFs). The latter were seeded onto coverslips and infected with adenovirus to express the Epac1-cAMP sensor (see above). The CM pellet was resuspended in $10 \mathrm{ml}$ buffer, then split into 2 aliquots, and centrifuged again ( 1 minute, $100 \mathrm{~g}$ ). Aliquots were resuspended in $500 \mu$ l buffer D (137 mmoles/l NaCl, 5.4 mmoles/l KCl, 2 mmoles/ $1 \mathrm{CaCl}_{2}, 1$ mmoles/1 $\mathrm{MgCl}_{2}, 10$ mmoles/l HEPES, pH, 7.3), one containing Iso $(10 \mu \mathrm{M})$, the other unsupplemented as a control. After incubation for 1 hour $\left(37^{\circ} \mathrm{C}, 5 \% \mathrm{CO}_{2}\right)$, cells were centrifuged and the conditioned media (Iso-coM and control-coM, respectively) were superfused to the aforementioned CFs during FRET recording. An alternative approach without intermediate cell centrifugation employed CMs and CFs from neonatal rat hearts: NRCMs were isolated from 1- to 2-day-old Sprague-Dawley rats as above and seeded $\left(1.5 \times 10^{6}\right.$ cells $/ 6 \mathrm{~cm}$ dish); after 24 hours, $300 \mu$ lof buffer $D$ was added, either including Iso $(10 \mu \mathrm{M})$ or without Iso addition. After 1 hour at $37^{\circ} \mathrm{C}, 5 \% \mathrm{CO}_{2}$, the Iso-coM and control-coM were collected and were superfused to the sensor CFs. For adenosine receptor antagonization, DPSPX (10 nM) was added to CFs 10 minutes before FRET measurement. For ABCC inhibition, MK571 $(50 \mu \mathrm{M})$ was added to CMs concomitant with the Iso-containing buffer $\mathrm{D}$ and, in addition, the basal medium of the CF culture was supplemented with MK571 at 10 minutes before measurements.

Signaling between myocardial slices and sensor CFs through secreted $c A M P$. CFs were isolated from $A d r b 1^{-/-} A d r b 2^{-/-}$mice and were infected with the Adv cAMP sensor for 2 days. Vital slices of myocardial tissue were prepared from 12-week-old mice according to an established protocol (22). Fresh slices were seeded with trypsinized sensor fibroblasts and were maintained in coculture for a further 12 hours under conditions providing an air-liquid interface (Millicell-CM, Millipore) (22). CFs were then trypsinized and seeded directly on the slices (prepared and incubated as described in ref. 22). After 12 hours, slices with adherent CFs were transferred to the experimental chamber in buffer $\mathrm{C}\left(137 \mathrm{mM} \mathrm{NaCl}, 5.4 \mathrm{mM} \mathrm{KCl}, 2 \mathrm{mM} \mathrm{CaCl}_{2}, 1 \mathrm{mM} \mathrm{MgCl}_{2}\right.$, $10 \mathrm{mM}$ HEPES, pH 7.3) and images were taken as described above. For contractility measurements, tissue slices from transversely cut mouse hearts were attached to organ hooks so that contraction forces of the septum and the lateral ventricular wall could be determined. Slices were mounted in a heated $\left(35^{\circ} \mathrm{C}\right)$ organ bath perfused with oxygenated $\left(95 \% \mathrm{O}_{2}, 5 \% \mathrm{CO}_{2}\right)$ Krebs-Ringer solution at $4 \mathrm{ml} / \mathrm{min}$. Electrical field stimulation (240 bpm, $1 \mathrm{~ms}$ pulse duration) was performed at 1.5 -fold excitation threshold (approximately $30 \mathrm{~mA}$ ). Preload was adjusted to 30 to $40 \mathrm{mg}$. Drugs were added to the perfusate by continuous infusion of 40 -fold concentrated stock solutions. Data were recorded with the software WinEDR (J. Dempster, University of Strathclyde, Glasgow, United Kingdom) and were quantified using the cyclic measurements function of LabChart (ADInstruments).

Ectonucleoside triphosphate diphosphohydrolase assays. Inhibitory activity at ectonucleoside triphosphate diphosphohydrolases (ENTPDs) was assayed at $37^{\circ} \mathrm{C}$ in a final volume of $100 \mu$ l. The reaction mixture contained $4 \mathrm{mM} \mathrm{CaCl}_{2}, 4 \mathrm{mM} \mathrm{MgCl}_{2}, 40 \mathrm{mM}$ HEPES, pH 7.4, and $400 \mu \mathrm{M}$ ATP as substrate. Solutions $(20 \mu \mathrm{l})$ of SYL-001 (various concentrations) in enzyme assay buffer were added, and the reaction was initiated by the addition of $20 \mu \mathrm{l}$ of human ENTPD1 (0.3 $\mu \mathrm{g})$, human ENTPD2 $(0.5 \mu \mathrm{g})$, or human ENTPD3 $(1.2 \mu \mathrm{g})$, respectively. The mixture was incubated for 30 minutes and terminated by heating at $99^{\circ} \mathrm{C}$ for 5 minutes. After cooling the reaction samples on ice, they were transferred into capillary electrphoresis (CE) vials and injected into the $\mathrm{CE}$ instrument. The operation conditions in $\mathrm{CE}$ analyses were as follows: all experiments were carried out using a P/ACE MDQ Capillary Electrophoresis System (Beckman Instruments) equipped with a DAD Detection System. Data collection and peak area analysis were performed by the P/ACE MDQ software 32 KARAT obtained from Beckman Coulter (Fullerton). The electrophoretic separations were carried out using a polyacrylamide-coated capillary $(60 \mathrm{~cm}[50 \mathrm{~cm}$ effective length], $\times 50 \mu \mathrm{m}$ [id]; obtained from CS-Chromatography). Electrokinetic injections were performed using a voltage of $-6 \mathrm{kV}$ for $60 \mathrm{sec}-$ onds, and separations were carried out by a voltage of $-20 \mathrm{kV}$. Analytes were detected using direct UV absorbance at $260 \mathrm{~nm}$. The capillary temperature was kept constant at $15^{\circ} \mathrm{C}$, and the temperature of the storing unit was adjusted to $15^{\circ} \mathrm{C}$. The running buffer consisted of 100 $\mathrm{mM}$ phosphate buffer ( $\mathrm{pH}$ 6.5). Between separations, the capillary was washed with water for 2 minutes (20 psi) and subsequently with running buffer for 2 minutes ( $20 \mathrm{psi}$ ) before each injection. All experiments were performed twice in triplicate. The $\mathrm{K}_{\mathrm{i}}$ values were determined by curve fitting of the data using Prism (GraphPad Software).

Ectonucleotide pyrophosphatase/phosphodiesterase assays. ENPP1 and ENPP3 inhibition assays were carried out at $37^{\circ} \mathrm{C}$ in a final volume of $100 \mu \mathrm{l}$. The reaction mixture contained $1 \mathrm{mM} \mathrm{MgCl}_{2}, 2 \mathrm{mM}$ $\mathrm{CaCl}_{2}, 10 \mathrm{mM}$ CHES, pH 9.0, and $400 \mu \mathrm{M}$ ATP as substrate. Solutions $(20 \mu \mathrm{l})$ of SYL-001 (various concentrations) in enzyme assay buffer were added, and the reaction was initiated by the addition of $20 \mu \mathrm{l}$ of human ENPP1 (1.7 $\mu \mathrm{g})$ or human ENPP3 (43 $\mu \mathrm{g})$, respectively. The mixture was incubated for 30 minutes (ENPP1) or 60 minutes (ENPP3), respectively, and terminated by heating at $90^{\circ} \mathrm{C}$ for $3 \mathrm{~min}-$ utes. After cooling the reaction samples on ice, they were transferred into $\mathrm{CE}$ vials and injected into the $\mathrm{CE}$ instrument. The operation conditions in CE analyses were the same as for ENTPDase assays. $\mathrm{K}_{\mathrm{i}}$ values were determined by curve fitting using Prism 5.0. Inhibition mechanisms were determined using 5 different concentrations of ATP (from 20 to $500 \mu \mathrm{M}$ ) and 3 different concentrations $(0,15$, and $60 \mathrm{nM}$ ) of inhibitor. All experiments were performed twice in triplicate. The inhibition type of each inhibitor was evaluated graphically from the Lineweaver-Burk plots using Prism.

ENPP2 assays were conducted at $37^{\circ} \mathrm{C}$ in a final volume of $50 \mu \mathrm{l}$. The reaction mixture contained $5 \mathrm{mM} \mathrm{MgCl}_{2}, 5 \mathrm{mM} \mathrm{CaCl}_{2}, 100 \mathrm{mM}$ Tris, pH 9.0, $400 \mu \mathrm{M}$ 1-oleoyl-sn-glycero-3-phosphocholine (LPC) (18:1) as substrate, and SYL-001 (different concentrations). The reaction was started by the addition of $10 \mu \mathrm{l}$ of human ENPP2 $(44 \mu \mathrm{g})$. The mixture was incubated at $37^{\circ} \mathrm{C}$ for 60 minutes, and subsequently, the released choline was quantified colorimetrically at $555 \mathrm{~nm}$ after incubation at $37^{\circ} \mathrm{C}$ for 10 minutes with $50 \mu \mathrm{l}$ of each of the following: the peroxidase reagent $(50 \mathrm{mM}$ Tris at $\mathrm{pH} 9.0,2 \mathrm{mM}$ TOOS, $5 \mathrm{U} / \mathrm{ml}$ peroxidase) and the choline oxidase reagent (50 mM Tris at $\mathrm{pH} 9.0$, $2 \mathrm{mM} 4$-aminoantipyrine, $5 \mathrm{U} / \mathrm{ml}$ choline oxidase). All experiments were performed twice in triplicate. The $\mathrm{K}_{\mathrm{i}}$ values were determined by curve fitting of the data using Prism 5.0.

NT5E. Evaluation of the inhibitory effect of SYL-001 on human NT5E activity was carried out in a mixture containing $4 \mathrm{mM} \mathrm{CaCl}$, $4 \mathrm{mM} \mathrm{MgCl}_{2}, 40 \mathrm{mM}$ HEPES, pH 7.4, $400 \mu \mathrm{M}$ AMP as substrate, and SYL-001 in different concentrations. The enzyme reaction was started by adding $0.11 \mu \mathrm{g}$ of recombinant human NT5E; then the mixture was incubated at $37^{\circ} \mathrm{C}$ for 30 minutes, and the reaction was subse- 
quently terminated by heating at $99^{\circ} \mathrm{C}$ for 5 minutes. Finally, $50 \mu \mathrm{l}$ of the reaction mixture was transferred into mini-CE vials containing $50 \mu \mathrm{l}$ of the internal standard uridine (final concentration, $6.25 \mu \mathrm{M}$ ). The operation conditions in CE were as follows: P/ACE MDQ capillary electrophoresis system, fused silica capillary $(40 \mathrm{~cm}[30 \mathrm{~cm}$ effective length], $\times 75.5 \mu \mathrm{m}$ (id); obtained from Polymicro Technologies), hydrodynamic injection (0.5 psi, $5 \mathrm{~s}$ ), separation voltage of 15 $\mathrm{kV}$, running buffer (40 mM borax and $100 \mathrm{mM}$ SDS at $\mathrm{pH} 9.0$ ), and detection at $260 \mathrm{~nm}$. Between separations, the capillary was washed with $0.1 \mathrm{~N}$ aqueous (aq.) $\mathrm{NaOH}$ solution for 2 minutes (30 psi) and subsequently with running buffer for 1 minute (30 psi) before each injection. All experiments were performed twice in triplicate. The $\mathrm{K}_{\mathrm{i}}$ values were determined by curve fitting of the data using Prism.

Tissue-nonspecific alkaline phosphatase assays. Reactions for tissue-nonspecific alkaline phosphatase (ALPL) inhibition studies were performed in a 96-well plate in a total volume of $100 \mu \mathrm{l}$. Assay buffer containing $1 \mathrm{mM} \mathrm{CaCl}_{2}, 2 \mathrm{mM} \mathrm{MgCl}, 10 \mathrm{mM}$ CHES, pH 10.5, various concentrations of SYL001, and $400 \mu \mathrm{M} p$-nitrophenyl phosphate as a substrate were used. The reaction was initiated with $0.12 \mu \mathrm{g}$ of human ALPL, and after 30 minutes of incubation at $37^{\circ} \mathrm{C}$, the liberated $p$-nitrophenolate was measured colorimetrically at $400 \mathrm{~nm}$. All experiments were performed twice in triplicate. The $\mathrm{K}_{\mathrm{i}}$ values were determined by curve fitting of the data using Prism 5.0.

Immunohistochemical analyses. The cross-sectional area in CMs was determined on $6-\mu \mathrm{m}$-thick paraffin-embedded tissue sections of left ventricular myocardium stained with Alexa Fluor 647-labeled wheat-germ agglutinin (WGA) (Life Technologies) to determine cell borders and SYTOX Green (Life Technologies) to detect nuclei. Images were taken from areas of transversely cut muscle fibers by confocal microscopy (Leica TCS SP5 II, $\times 20$ objective; laser lines, $488 \mathrm{~nm}$ for SYTOX Green and $633 \mathrm{~nm}$ for WGA). Individual cells were analyzed in an automated manner using morphology filters of MetaMorph software (Molecular Devices) to draw lines separating individual cells based on WGA staining and to exclude cells with nuclei touching a cell border. Thresholding was applied to exclude regions of background (no cells) or extensive fibrosis. The average area of myocytes with centralized nucleus in 1 section $(n=59-200)$ was calculated using the MetaMorph integrated morphometry analysis function. Apoptosis was assessed by TUNEL staining using the In Situ Cell Death Detection Kit, TMR Red (Roche), according to the manufacturer's instructions for treatment of paraffin-embedded tissue. Briefly, sections of paraffin-preserved mouse hearts (6-8 $\mu \mathrm{m})$ were rehydrated, followed by 5 minutes of microwave irradiation in antigen-retrieval solution (Dako). The samples were incubated with TUNEL reaction mixture for 1 hour at $37^{\circ} \mathrm{C}$ in the dark. SYTOX Green (Life Technologies) was added for nuclear counterstaining. Both a negative control (without enzyme solution) and a positive control (pretreatment with DNase for 10 minutes at room temperature) were included. Images of whole-heart sections were acquired by a confocal microscope ( $\times 20$ objective, laser lines, 488 $\mathrm{nm}$ for SYTOX Green and $561 \mathrm{~nm}$ for TMR). Red (TUNEL positive) and green (210,000-340,000 per group) nuclei were automatically counted using an image analysis algorithm (MetaMorph). To visualize the embedding of sensor CFs into myocardial slices, tissue was fixed in $4 \%$ PFA overnight at $4^{\circ} \mathrm{C}$. After permeabilization with $1 \%$ Triton-X 100 for 30 minutes at room temperature and RNAse treatment $\left(100 \mu \mathrm{g} / \mathrm{ml}, 20\right.$ minutes, $\left.37^{\circ} \mathrm{C}\right)$, slices were stained overnight at $4^{\circ} \mathrm{C}$ with Alexa Fluor 647 phalloidin (1:100, Life Technologies), followed by 2 hours incubation at room temperature with secondary antibody Alexa Fluor 488 goat anti-rabbit (Life Technologies). Nuclei were stained with propidium iodide (1:200, Life Technologies) for 15 minutes at $37^{\circ} \mathrm{C}$. Slices were mounted in Aquatex (Mer$\mathrm{ck})$, and confocal imaging was performed.

Quantitative real-time PCR. Total RNA was prepared with RNeasy Mini Kits (QIAGEN), and 500 ng was reverse transcribed using a standard protocol (Superscript II, Invitrogen). Quantitative real-time PCR amplification of adenosine receptor mRNA was performed with the primers listed below, using the FastStart universal SYBR Green Master Mix (Roche). The specificity of each primer set was monitored by analyzing the dissociation curve. The sample volume was $12.5 \mu \mathrm{l}$, containing $1 \times$ SYBR Green Master Mix, $400 \mathrm{nM}$ gene-specific primers, and a $2.5 \mu \mathrm{l}$ template. Sequences of primers used for real-time PCR were as follows (gene symbols and species followed by sequences of forward and reverse primers): Adora1 rat (5'-ATTGCTGTGGATCGATACC-3'， 5'-GAATCCAGCAGCCAGCTAT-3'); Adora $2 a$ rat (5'-GCAGAGTTCCATCTTTAGC-3', 5'-CGCCCTCACACCTGTCA-3'); Adora2b rat (5'-TCCATCTTTAGCCTCTTGG-3', 5'-TCCTCTTGCTCGTGTTC-3'); Adora3 rat (5'-CTGCGAGTCAAGCTGAC-3', 5'-GTCCCACCAGAAAGGACA-3'); Abcc4 rat (5'-CAACAGAAGATCCGGGAGAA-3', 5'-TTCTGCAGCAAGACATACGG-3'); Gapdh rat (5'-TGACAACTCCCTCAAGATTGTCA-3', 5'-GGCATGGACTGTGGTCATGA-3'); RPL32 rat (5'-TCTGGTCCACAATGTCAAGG-3', 5'-TGTGCTGCTCTTTCTACGATG-3'); Colla2 mouse (5'-AGGTCTTCCTGGAGCTGATG-3'， 5'-ACCCACAGGGCCTTCTTTAC-3'); Col3a1 mouse (5'-ACAGCAAATTCACTTACACAGTTC-3', 5'-CTCATTGCCTTGCGTGTTT-3'); Myh7 mouse (5'-ACTGTCAACACTAAGAGGGTCA-3', 5'-TTGGATGATTTGATCTTCCAGGG-3'); Adora1 mouse (5'-GTGATTTGGGCTGTGAAGGT-3', 5'-AGTAGGTCTGTGGCCCAATG-3'); Adora2a mouse (5'-TGCAGAACGTCACCAACTTC-3', 5'-CAAAACAGGCGAAGAAGAGG-3'); Adora $2 b$ mouse (5'-GGCTATGATTGTGGGCATCT-3', 5'-GACAACTGAATTGGCGTGTG-3'); Adora3 mouse (5'-TCCCTGATTACCACGGACTC-3', 5'-TCCTTCTGTTCCCCACATTC-3'); and Gapdh mouse (5'-GTGAAGGTCGGTGTGAACG-3', 5'-TCGTTGATGGCAACAATCTC-3').

Statistics. All quantitative data are reported as mean \pm SEM. Statistical analysis was performed with the Prism software package (GraphPad version 6). Data distribution was assessed by a ShapiroWilk test for normality. Common variance was tested using the F-test or Bartlett's test. Differences between 2 means were assessed by a 2-tailed paired or unpaired $t$ test. Differences among multiple means were assessed, as indicated, by 1-way or 2-way ANOVA followed by Bonferroni's test analysis. If the sample number did not suffice to test for normality (<8 per group), nonparametric tests (Mann-Whitney $U$ test or 1-way ANOVA followed by Holm-Sidak's test analysis) were used. A $P$ value of less than 0.05 was considered significant.

Study approval. Animal care and experimental procedures were approved by the local authorities (Regierung von Oberbayern, Munich, Germany).

\section{Acknowledgments}

We thank Isabell Flohrschütz, Lucia Koblitz, and Urszula Kremser for primary cell isolations; Sabine Brummer for performing 
cardiac histology; Monika Brill and Thomas Misgeld for help with confocal microscopy; John Schuetz for providing the Abcc4-deficient mice; and Ken Jacobson for technical advice. We are grateful to Tom Schwarzer for his help with the FRET assays. This work was supported in part by grants from the Bundesministerium für Bildung und Forschung (BMBF); by the Bavarian Ministry of Sciences, Research and the Arts in the framework of the Bavarian
Molecular Biosystems Research Network; and by the Bayerische Forschungsstiftung (postdoctoral fellowship for Y. Sassi).

Address for correspondence: Stefan Engelhardt, Institut für Pharmakologie und Toxikologie, Technische Universität München (TUM), Biedersteiner Straße 29, 80802 Munich, Germany. Phone: 49.89.4140.3260; E-mail: stefan.engelhardt@tum.de.
1. Lefkowitz RJ, Rockman HA, Koch WJ. Catecholamines, cardiac $\beta$-adrenergic receptors, and heart failure. Circulation. 2000;101(14):1634-1637.

2. Lohse MJ, Engelhardt S, Eschenhagen T. What is the role of beta-adrenergic signaling in heart failure? Circ Res. 2003;93(10):896-906.

3. Choi DJ, Rockman HA. $\beta$-Adrenergic receptor desensitization in cardiac hypertrophy and heart failure. Cell Biochem Biophys. 1999;31(3):321-329.

4. Van Aubel RA, Smeets PH, Peters JG, Bindels RJ, Russel FG. The MRP4/ABCC4 gene encodes a novel apical organic anion transporter in human kidney proximal tubules: putative efflux pump for urinary cAMP and cGMP. J Am Soc Nephrol. 2002;13(3):595-603.

5. Chen ZS, Lee K, Kruh GD. Transport of cyclic nucleotides and estradiol 17- $\beta$-D-glucuronide by multidrug resistance protein 4 . Resistance to 6-mercaptopurine and 6-thioguanine. J Biol Chem. 2001;276(36):33747-33754.

6. Jedlitschky G, Burchell B, Keppler D. The multidrug resistance protein 5 functions as an ATPdependent export pump for cyclic nucleotides. J Biol Chem. 2000;275(39):30069-30074.

7. Guo Y, et al. MRP8, ATP-binding cassette C11 (ABCC11), is a cyclic nucleotide efflux pump and a resistance factor for fluoropyrimidines 2',3'-dideoxycytidine and 9'-(2'-phosphonylmethoxyethyl)adenine. J Biol Chem. 2003;278(32):29509-29514.

8. Ahloulay M, Déchaux M, Hassler C, Bouby N, Bankir L. Cyclic AMP is a hepatorenal link influencing natriuresis and contributing to glucagoninduced hyperfiltration in rats. JClin Invest. 1996;98(10):2251-2258.

9. McIntosh VJ, Lasley RD. Adenosine receptormediated cardioprotection: are all 4 subtypes required or redundant? J Cardiovasc Pharmacol Ther. 2012;17(1):21-33.

10. Jackson EK, Raghvendra DK. The extracellular cyclic AMP-adenosine pathway in renal physiology. Annu Rev Physiol. 2004;66:571-599.

11. Sassi Y, et al. Regulation of cAMP homeostasis by the efflux protein MRP4 in cardiac myocytes. FASEB J. 2012;26(3):1009-1017.

12. Dubey RK, Gillespie DG, Mi Z, Jackson EK. Endogenous cyclic AMP-adenosine pathway regulates cardiac fibroblast growth. Hypertension. 2001;37(4):1095-1100.

13. Goding JW, Grobben B, Slegers H. Physiological and pathophysiological functions of the ectonucleotide pyrophosphatase/phosphodiesterase family. Biochim Biophys Acta. 2003;1638(1):1-19.

14. Koszalka P, et al. Targeted disruption of cd73/ ecto-5'-nucleotidase alters thromboregulation and augments vascular inflammatory response. Circ Res. 2004;95(8):814-821.

15. Xu X, et al. Ecto-5'-nucleotidase deficiency exac- erbates pressure-overload-induced left ventricular hypertrophy and dysfunction. Hypertension. 2008;51(6):1557-1564.

16. Eckle T, et al. Cardioprotection by ecto-5'nucleotidase (CD73) and $\mathrm{A} 2 \mathrm{~B}$ adenosine receptors. Circulation. 2007;115(12):1581-1590.

17. Weyler S, et al. Improving potency, selectivity, and water solubility of adenosine A1 receptor antagonists: xanthines modified at position 3 and related pyrimido[1,2,3-cd]purinediones. ChemMedChem. 2006;1(8):891-902.

18. Müller CE, Jacobson KA. Recent developments in adenosine receptor ligands and their potential as novel drugs. Biochim Biophys Acta. 2011;1808(5):1290-1308.

19. Epperson SA, Brunton LL, Ramirez-Sanchez I, Villarreal F. Adenosine receptors and second messenger signaling pathways in rat cardiac fibroblasts. Am J Physiol Cell Physiol. 2009;296(5):C1171-C1177.

20. Rohrer D, Chruscinski A, Schauble E, Bernstein D, Kobilka B. Cardiovascular and metabolic alterations in mice lacking both $\beta 1$ - and $\beta 2$-adrenergic receptors. J Biol Chem. 1999;274(24):16701-16708.

21. Bera T, Lee S, Salvatore G, Lee B, Pastan I. MRP8, a new member of $A B C$ transporter superfamily, identified by EST database mining and gene prediction program, is highly expressed in breast cancer. Mol Med. 2001;7(8):509-516.

22. Brandenburger M, et al. Organotypic slice culture from human adult ventricular myocardium. Cardiovasc Res. 2012;93(1):50-59.

23. Liao Y, et al. Activation of adenosine A1 receptor attenuates cardiac hypertrophy and prevents heart failure in murine left ventricular pressureoverload model. Circ Res. 2003;93(8):759-766.

24. Marienfeld U, Walter U, Simm A. Inhibition of rat cardiac fibroblast growth by cAMP - but not by cGMP-dependent protein kinase. Basic Res Cardiol. 2001;96(2):184-191.

25. Brundege J, Diao L, Proctor W, Dunwiddie T. The role of cyclic AMP as a precursor of extracellular adenosine in the rat hippocampus. Neuropharmacology. 1997;36(9):1201-1210.

26. Chiavegatti T, Costa V Jr, Araujo M, Godinho R. Skeletal muscle expresses the extracellular cyclic AMP - adenosine pathway. Br J Pharmacol. 2008;153(6):1331-1340.

27. Carey GB, Wotjukiewicz LJ, Goodman JM, Reineck KE, Overman KC. Extracellular cyclic $\mathrm{AMP}$ and adenosine appearance in adipose tissue of sus scrofa: effects of exercise. Exp Biol Med. 2004;229(10):1026-1032.

28. Corriden R, Insel P. Basal release of ATP: an autocrine-paracrine mechanism for cell regulation. Sci Signal. 2010;3(104):re1.

29. Sager G. Cyclic GMP transporters. Neurochem Int.
2004;45(6):865-873

30. Gödecke S, Stumpe T, Schiller H, Schnittler HJ, Schrader J. Do rat cardiac myocytes release ATP on contraction? Am J Physiol Cell Physiol. 2005;289(3):C609-C616.

31. Lu D, Insel PA. Hydrolysis of extracellular ATP by ectonucleoside triphosphate diphosphohydrolase (ENTPD) establishes the set point for fibrotic activity of cardiac fibroblasts. J Biol Chem. 2013;288(26):19040-19049.

32. Toufektsian M, et al. Stimulation of A2Aadenosine receptors after myocardial infarction suppresses inflammatory activation and attenuates contractile dysfunction in the remote left ventricle. Am J Physiol Heart Circ Physiol. 2006;290(4):H1410-H1418.

33. Yang D, et al. The A2b adenosine receptor protects against vascular injury. Proc Natl Acad Sci US A. 2008;105(2):792-796.

34. Wakeno M, et al. Long-term stimulation of adenosine $\mathrm{A} 2 \mathrm{~b}$ receptors begun after myocardial infarction prevents cardiac remodeling in rats. Circulation. 2006;114(18):1923-1932.

35. Eltzschig HK, Bonney SK, Eckle T. Attenuating myocardial ischemia by targeting A2B adenosine receptors. Trends Mol Med. 2013;19(6):345-354.

36. Meyer TE, et al. Antiadrenergic effects of adenosine in pressure overload hypertrophy. Hypertension. 2001;37(3):862-868.

37. Yang Z, et al. Cardiac overexpression of A1-adenosine receptor protects intact mice against myocardial infarction. Am J Physiol Heart Circ Physiol. 2002;282(3):H949-H955.

38. Dubey RK, Gillespie DG, Mi Z, Jackson EK. Cardiac fibroblasts express the cAMP-adenosine pathway. Hypertension. 2000;36(3):337-342.

39. Jacobson K, Gao Z. Adenosine receptors as therapeutic targets. Nat Rev Drug Discov. 2006;5(3):247-264.

40. Hofer AM, Lefkimmiatis K. Extracellular calcium and CAMP: second messengers as "third messengers"? Physiology (Bethesda, Md). 2007;22:320-327.

41. Muller C. General synthesis and properties of 1-monosubstituted xanthines. Synthesis. 1993;1:125-128.

42. Sauer R, et al. Water-soluble phosphate prodrugs of 1-propargyl-8-styrylxanthine derivatives, $\mathrm{A}(2 \mathrm{~A})$-selective adenosine receptor antagonists. JMed Chem. 2000;43(3):440-448.

43. Hockemeyer J, Burbiel JC, Muller CE. Multigram-scale syntheses, stability, and photoreactions of $\mathrm{A} 2 \mathrm{~A}$ adenosine receptor antagonists with 8-styrylxanthine structure: potential drugs for parkinson's disease. JOrg Chem. 2004;69(10):3308-3318.

44. Rockman HA, et al. Segregation of atrial-specific and inducible expression of an atrial natriuretic factor transgene in an in vivo murine model of 
cardiac hypertrophy. Proc Natl Acad Sci U S A. 1991;88(18):8277-8281.

45. Leggas M, et al. Mrp4 confers resistance to topotecan and protects the brain from chemotherapy. Mol Cell Biol. 2004;24(17):7612-7621.

46. De Wolf CJF, et al. cGMP transport by vesicles from human and mouse erythrocytes. FEBS $J$. 2007;274(2):439-450.
47. Frost RJA, Engelhardt S. A secretion trap screen in yeast identifies protease inhibitor 16 as a novel antihypertrophic protein secreted from the heart. Circulation. 2007;116(16):1768-1775.

48. Jentzsch C, et al. A phenotypic screen to identify hypertrophy-modulating microRNAs in primary cardiomyocytes. J Mol Cell Cardiol. 2012;52(1):13-20.
49. Bang C, et al. Cardiac fibroblast - derived microRNA passenger strand-enriched exosomes mediate cardiomyocyte hypertrophy. JClin Invest. 2014;124(5):2136-2146.

50. Nikolaev VO, Bünemann M, Hein L, Hannawacker A, Lohse MJ. Novel single chain cAMP sensors for receptor-induced signal propagation. J Biol Chem. 2004;279(36):37215-37218. 